\title{
Low-energy effects of Lepton Flavour Universality Violation
}

\author{
Claudia Cornella, ${ }^{a}$ Ferruccio Feruglio ${ }^{b}$ and Paride Paradisi $^{b}$ \\ ${ }^{a}$ Physik-Institut, Universität Zürich, \\ CH-8057 Zürich, Switzerland \\ ${ }^{b}$ Dipartimento di Fisica e Astronomia 'G. Galilei', Università di Padova, \\ INFN, Sezione di Padova, \\ Via Marzolo 8, I-35131 Padua, Italy \\ E-mail: claudia.cornella@physik.uzh.ch, feruglio@pd.infn.it, \\ paradisi@pd.infn.it
}

ABSTRACT: The persisting anomalous data in semileptonic B-decays point towards New Physics models exhibiting large sources of Lepton Flavour Universality Violation. In this work we generalise previous studies by considering frameworks which include an enlarged set of semileptonic four-fermion operators invariant under the SM gauge group, with New Physics affecting mainly the third generation. We derive the low-energy effective Lagrangian including the leading electroweak corrections, mandatory to obtain reliable predictions. As a particularly interesting case, we analyse the scenario where the dominant New Physics effects are encoded in the Wilson coefficient $C_{9}$, as favoured by global fit analyses of $b \rightarrow s$ data. We find that also in this case the stringent experimental bounds on $Z$-pole observables and $\tau$ decays challenge a simultaneous explanation of charged and neutral-current non-standard data.

KeYwords: Beyond Standard Model, Heavy Quark Physics

ARXIV EPRINT: 1803.00945 


\section{Contents}

1 Introduction 1

2 Theoretical framework 3

3 Observables $\quad 8$

3.1 The $B$ anomalies 12

3.1.1 $B \rightarrow K \ell \bar{\ell} \quad 13$

3.1.2 $B \rightarrow D^{(*)} \ell \nu \quad 13$

$\begin{array}{lll}3.2 & \text { Tree-level semileptonic phenomenology } & 14\end{array}$

3.2.1 $B \rightarrow \ell \nu \quad 14$

$\begin{array}{ll}3.2 .2 \quad B \rightarrow K^{(*)} \nu \bar{\nu} & 14\end{array}$

$\begin{array}{lll}3.2 .3 \quad B_{s} \rightarrow \mu \bar{\mu} & 15\end{array}$

$\begin{array}{lll}3.2 .4 & \text { Lepton-flavour violating } B \text { decays } & 15\end{array}$

$\begin{array}{lll}3.3 & \text { One-loop phenomenology } & 15\end{array}$

$\begin{array}{lll}\text { 3.3.1 } & Z \text {-pole observables } & 15\end{array}$

$\begin{array}{lll}\text { 3.3.2 } & \text { Purely leptonic effective Lagrangian } & 16\end{array}$

$\begin{array}{lll}3.3 .3 \tau \rightarrow \ell \bar{\nu} \nu & 17\end{array}$

$\begin{array}{lll}3.3 .4 & \tau \rightarrow 3 \mu & 18\end{array}$

$\begin{array}{lll}3.4 & \text { Numerical analysis } & 18\end{array}$

4 Conclusions $\quad 21$

\section{Introduction}

In the last few years, various experimental collaborations observed indications of Lepton Flavour Universality Violation (LFUV) in semileptonic $B$ decays. Although such indications are not yet conclusive, the overall pattern of deviations from the Standard Model (SM) predictions is very coherent. The anomalous data refer to i) charged-current transitions $b \rightarrow c \ell \bar{\nu}$ with $\tau / e$ and $\tau / \mu$ LFUV [1-4] and ii) neutral-current transitions $b \rightarrow s \ell \bar{\ell}$ with $\mu / e$ LFUV $[5,6]$. Interestingly enough, global fit analyses for the angular distributions of the $B^{0} \rightarrow K^{* 0} \mu^{+} \mu^{-}$decay reported anomalies which are consistent with LFUV data $[7-9]$.

From a theoretical point of view, it would be desirable to explain both the chargedand neutral-current anomalies within a coherent extension of the SM [10-19]. A first step towards this goal is represented by an effective theory where the effects of New Physics (NP) are described by four-fermion operators involving left-handed currents, $\left(\bar{s}_{L} \gamma_{\mu} b_{L}\right)\left(\bar{\mu}_{L} \gamma_{\mu} \mu_{L}\right)$ and $\left(\bar{c}_{L} \gamma_{\mu} b_{L}\right)\left(\bar{\tau}_{L} \gamma_{\mu} \nu_{L}\right)$, which are related by the $\mathrm{SU}(2)_{L}$ gauge symmetry [20,21]. A crucial ingredient of such a theory requires that NP couples much more strongly to the third 
generation than to the first two, since $\left(\bar{c}_{L} \gamma_{\mu} b_{L}\right)\left(\bar{\tau}_{L} \gamma_{\mu} \nu_{L}\right)$ is induced already at the tree level in the SM while $\left(\bar{s}_{L} \gamma_{\mu} b_{L}\right)\left(\bar{\mu}_{L} \gamma_{\mu} \mu_{L}\right)$ arises only at loop-level. The latter requirement is realized, for instance, if NP is coupled only to the third fermion generation in the interaction basis. Couplings to lighter generations are generated after electroweak symmetry breaking by the misalignment between the mass and the interaction bases through small flavour mixing angles [22].

Hence, a minimal framework addressing the B-anomalies consists of an effective Lagrangian defined above the electroweak scale and containing gauge-invariant semileptonic operators involving purely left-handed fermions of the third generation. Assuming such starting point, in $[23,24]$ the low-energy effective Lagrangian including leading electroweak corrections was derived. The most striking effects found were large corrections to the leptonic couplings of the $W$ and $Z$ vector bosons and the generation of a purely leptonic effective Lagrangian. The resulting LFUV in $Z$ and $\tau$ decays and $\tau$ Lepton Flavour Violating (LFV) contributions turned out to challenge a simultaneous explanation of chargedand neutral-current anomalies. Although this conclusion applies under certain assumptions and possible ways-out have been already identified in the literature [25], our main message was that including electroweak corrections is mandatory when addressing the B-anomalies with $\mathrm{NP}$ at the $\mathrm{TeV}$ scale. Another important challenge that one has to face is the lack of signals in direct production at LHC of any mediators responsible of the four-fermion interactions invoked to explain the B-anomalies [25-27].

In this paper we make a step forward compared to [23, 24]. In particular, we consider both purely left-handed operators $(V-A) \times(V-A)$ as well as operators with right-handed currents of the form $(V+A) \times(V+A)$ and $(V \pm A) \times(V \mp A)$. This effort is justified by the fact that many NP models, proposed to accommodate B-anomalies, exhibit the operators considered here. ${ }^{1}$ For instance models with heavy mediators such as extra gauge vector bosons or scalar/vector leptoquarks can generate different combinations of the operators analysed here. Moreover, as we will discuss in the following, such enlarged operator basis will allow us to consider one of the most favoured solutions to the neutral-current anomalies, with dominant NP effects encoded in the low-energy Wilson coefficient $C_{9}$ [7-9]. While this solution is only mildly preferred over the one with $C_{9}=-C_{10}$ when explaining the neutral-current anomalies, it might be favoured when including the constraints coming from $Z$ and $\tau$ decays. The infrared behaviour of a scenario with the $O_{9}$ operator at the $\mathrm{TeV}$ scale can in principle be quite different from the one related to $C_{9}=-C_{10}$ and deserves a quantitative analysis to establish whether it can evade the bounds associated to purely leptonic processes or not. The paper is organised as follows. In section 2, we present the theoretical framework and construct the low-energy effective Lagrangian including electroweak corrections in the leading logarithm approximation. In section 3 , we examine the phenomenological implications of our setup, discussing both tree-level and loop-induced low-energy observables. Moreover, we assess the impact of these constraints

\footnotetext{
${ }^{1}$ We do not consider operators of scalar or tensor type [28-31]. The former are severely constrained by the $B_{c}$ lifetime through the enhancement of the $B_{c}^{-} \rightarrow \tau^{-} \bar{\nu}$ channel [28]. Renormalization of scalar and tensor operators, their strong mixing and the impact on phenomenology has been recently analysed in ref. [29].
} 
by means of a global numerical analysis. After discussing the scenario where the dominant NP effects are encoded in the Wilson coefficient $C_{9}$, we extend the analysis to the full parameter space. Our conclusions are presented in section 4.

\section{Theoretical framework}

We assume that strong and electroweak interactions at the scale $\Lambda \gg m_{W}$ are described by the effective Lagrangian

$$
\mathcal{L}=\mathcal{L}_{\mathrm{SM}}+\mathcal{L}_{\mathrm{NP}}^{0},
$$

where the NP contribution is given by

$$
\mathcal{L}_{\mathrm{NP}}^{0}=\frac{1}{\Lambda^{2}}\left(C_{1}\left[Q_{\ell q}^{(1)}\right]_{3333}+C_{3}\left[Q_{\ell q}^{(3)}\right]_{3333}+C_{4}\left[Q_{\ell d}\right]_{3333}+C_{5}\left[Q_{e d}\right]_{3333}+C_{6}\left[Q_{q e}\right]_{3333}\right)
$$

and the semileptonic operators $Q_{i}$ are defined in table 1, where primed fields indicate fields in the interaction basis. We denote the Wilson coefficients at the scale $\Lambda$ by $C_{1}=\left[\mathcal{C}_{\ell q}^{(1)}(\Lambda)\right]_{3333}, C_{3}=\left[\mathcal{C}_{\ell q}^{(3)}(\Lambda)\right]_{3333}$ and so on. Notice that (2.2) assumes that NP couples only to third generation fermions. Couplings to light generations will arise when switching from the interaction to the mass basis after electroweak symmetry breaking, as we will describe shortly. Such an assumption is motivated by the need of generating a hierarchy between NP effects in charged- and neutral-current semileptonic B-decays, as suggested by experimental data. Dominance of NP couplings to the third generation is a common feature of most of the current flavour models, such as those based on flavour symmetries or appealing to Minimal Flavour Violation or exploiting the partial compositeness scenario. While our assumption concerning the flavour pattern of NP reflects the properties of the existing models, it is evident that does not cover all conceivable possibilities. Our purpose is to define a simple baseline framework whose predictions can be compared with the experimental data. Later on we will discuss the departures from the baseline. We move to the mass basis, denoted by unprimed fields, by means of the unitary transformations

$$
\begin{array}{lll}
u_{L}^{\prime}=V_{u} u_{L} & d_{L}^{\prime}=V_{d} d_{L} & \ell_{L}^{\prime}=V_{e} \ell_{L}, \\
u_{R}^{\prime}=R_{u} u_{R} & d_{R}^{\prime}=R_{d} d_{R} & e_{R}^{\prime}=R_{e} e_{R},
\end{array}
$$

where we work in the approximation of massless neutrinos. To keep track of the flavour structure of the Lagrangian, we define the following matrices in flavour space

$$
\begin{aligned}
& \lambda_{i j}^{u}=V_{u 3 i}^{*} V_{u 3 j} \quad \lambda_{i j}^{d}=V_{d 3 i}^{*} V_{d 3 j} \quad \lambda_{i j}^{e}=V_{e 3 i}^{*} V_{e 3 j} \quad \lambda_{i j}^{u d}=V_{u 3 i}^{*} V_{d 3 j} \\
& \Gamma_{i j}^{d}=R_{d 3 i}^{*} R_{d 3 j} \quad \Gamma_{i j}^{e}=R_{e 3 i}^{*} R_{e 3 j},
\end{aligned}
$$

where $\lambda$ and $\Gamma$ are both projectors with trace equal to one, and the $\lambda$ matrices are related by $\lambda^{u}=V_{\mathrm{CKM}} \lambda^{d} V_{\mathrm{CKM}}^{\dagger}$ and $\lambda^{u d}=V_{\mathrm{CKM}} \lambda^{d}, V_{\mathrm{CKM}}=V_{u}^{\dagger} V_{d}$ being the quark mixing matrix. Hereafter we will omit the subscript CKM for simplicity. In the mass basis the Lagrangian 


\begin{tabular}{|l|c|c|c|}
\hline \multicolumn{2}{|c|}{ Leptonic operators } & \multicolumn{2}{|c|}{ Semileptonic operators } \\
\hline$\left[Q_{\ell \ell}\right]_{p r s t}$ & $\left(\bar{\ell}_{p L}^{\prime} \gamma_{\mu} \ell_{r L}^{\prime}\right)\left(\bar{\ell}_{s R}^{\prime} \gamma^{\mu} \ell_{t R}^{\prime}\right)$ & {$\left[Q_{\ell q}^{(1)}\right]_{p r s t}$} & $\left(\bar{\ell}_{p L}^{\prime} \gamma_{\mu} \ell_{r L}^{\prime}\right)\left(\bar{q}_{s L}^{\prime} \gamma^{\mu} q_{t L}^{\prime}\right)$ \\
{$\left[Q_{\ell e}\right]_{p r s t}$} & $\left(\bar{\ell}_{p L}^{\prime} \gamma^{\mu} \ell_{r L}^{\prime}\right)\left(\bar{e}_{s R}^{\prime} \gamma_{\mu} e_{t R}^{\prime}\right)$ & {$\left[Q_{\ell q}^{(3)}\right]_{p r s t}$} & $\left(\bar{\ell}_{p L}^{\prime} \gamma_{\mu} \tau^{a} \ell_{r L}^{\prime}\right)\left(\bar{q}_{s L}^{\prime} \gamma^{\mu} \tau^{a} q_{t L}^{\prime}\right)$ \\
{$\left[Q_{e e}\right]_{p r s t}$} & $\left(\bar{e}_{p R}^{\prime} \gamma_{\mu} e_{r R}^{\prime}\right)\left(\bar{e}_{s R}^{\prime} \gamma^{\mu} e_{t R}^{\prime}\right)$ & {$\left[Q_{\ell u}\right]_{p r s t}$} & $\left(\bar{\ell}_{p L}^{\prime} \gamma_{\mu} \ell_{r L}^{\prime}\right)\left(\bar{u}_{s R}^{\prime} \gamma^{\mu} u_{t R}^{\prime}\right)$ \\
& & {$\left[Q_{\ell d}\right]_{p r s t}$} & $\left(\bar{\ell}_{p L}^{\prime} \gamma_{\mu} \ell_{r L}^{\prime}\right)\left(\bar{d}_{s R}^{\prime} \gamma^{\mu} d_{t R}^{\prime}\right)$ \\
& & {$\left[Q_{q e}\right]_{p r s t}$} & $\left(\bar{q}_{p L}^{\prime} \gamma_{\mu} q_{r L}^{\prime}\right)\left(\bar{e}_{s R}^{\prime} \gamma^{\mu} e_{t R}^{\prime}\right)$ \\
& & {$\left[Q_{e u}\right]_{p r s t}$} & $\left(\bar{e}_{p R}^{\prime} \gamma_{\mu} e_{r R}^{\prime}\right)\left(\bar{u}_{s R}^{\prime} \gamma^{\mu} u_{t R}^{\prime}\right)$ \\
& & {$\left[Q_{e d}\right]_{p r s t}$} & $\left(\bar{e}_{p R}^{\prime} \gamma_{\mu} e_{r R}^{\prime}\right)\left(\bar{d}_{s R}^{\prime} \gamma^{\mu} d_{t R}^{\prime}\right)$ \\
\hline$\left[Q_{H \ell}^{(1)}\right]_{p r}$ & $\left(\phi^{\dagger} i \overleftrightarrow{D_{\mu}} \phi\right)\left(\bar{\ell}_{p L}^{\prime} \gamma^{\mu} \ell_{r L}^{\prime}\right)$ & {$\left[Q_{q q}^{(1)}\right]_{p r s t}$} & $\left(\bar{q}_{p L}^{\prime} \gamma_{\mu} q_{r L}^{\prime}\right)\left(\bar{q}_{s L}^{\prime} \gamma^{\mu} q_{t L}^{\prime}\right)$ \\
{$\left[Q_{H \ell}^{(3)}\right]_{p R}$} & $\left(\phi^{\dagger} i \overleftrightarrow{D_{\mu}^{a}} \phi\right)\left(\bar{\ell}_{p L}^{\prime} \gamma^{\mu} \tau^{a} \ell_{r L}^{\prime}\right)$ & {$\left[Q_{q q}^{(3)}\right]_{p r s t}$} & $\left(\bar{q}_{p L}^{\prime} \gamma_{\mu} \tau^{a} q_{r L}^{\prime}\right)\left(\bar{q}_{s L}^{\prime} \gamma^{\mu} \tau^{a} q_{t L}^{\prime}\right)$ \\
{$\left[Q_{H q}^{(1)}\right]_{p R}$} & $\left(\phi^{\dagger} i \overleftrightarrow{D_{\mu}} \phi\right)\left(\bar{q}_{p L}^{\prime} \gamma^{\mu} q_{r L}^{\prime}\right)$ & {$\left[Q_{q u}^{(1)}\right]_{p r s t}$} & $\left(\bar{q}_{p L}^{\prime} \gamma_{\mu} q_{r L}^{\prime}\right)\left(\bar{u}_{s R}^{\prime} \gamma^{\mu} u_{t R}^{\prime}\right)$ \\
{$\left[Q_{H q}^{(3)}\right]_{p R}$} & $\left(\phi^{\dagger} i \overleftrightarrow{D_{\mu}^{a}} \phi\right)\left(\bar{q}_{p L}^{\prime} \gamma^{\mu} \tau^{a} q_{r L}^{\prime}\right)$ & {$\left[Q_{q d}^{(1)}\right]_{p r s t}$} & $\left(\bar{q}_{p L}^{\prime} \gamma_{\mu} q_{r L}^{\prime}\right)\left(\bar{d}_{s R}^{\prime} \gamma^{\mu} d_{t R}^{\prime}\right)$ \\
{$\left[Q_{H e}\right]_{p R}$} & $\left(\phi^{\dagger} i \overleftrightarrow{D_{\mu}} \phi\right)\left(\bar{e}_{p R}^{\prime} \gamma_{\mu} e_{r R}^{\prime}\right)$ & {$\left[Q_{d d}\right]_{p r s t}$} & $\left(\bar{d}_{p R}^{\prime} \gamma_{\mu} d_{r R}^{\prime}\right)\left(\bar{d}_{s R}^{\prime} \gamma^{\mu} d_{t R}^{\prime}\right)$ \\
{$\left[Q_{H d}\right]_{p R}$} & $\left(\phi^{\dagger} i \overleftrightarrow{D_{\mu}} \phi\right)\left(\bar{d}_{p R}^{\prime} \gamma^{\mu} d_{r R}^{\prime}\right)$ & {$\left[Q_{u d}^{(1)}\right]_{p r s t}$} & $\left(\bar{u}_{p R}^{\prime} \gamma_{\mu} u_{r R}^{\prime}\right)\left(\bar{d}_{s R}^{\prime} \gamma^{\mu} d_{t R}^{\prime}\right)$ \\
\hline
\end{tabular}

Table 1. $\mathrm{SU}(2)_{L} \times \mathrm{U}(1)_{\mathrm{Y}}$ invariant operators involved in the renormalization group evolution of $\mathcal{L}_{\mathrm{NP}}^{0}$ from $\Lambda$ to the $\mathrm{EW}$ scale. We adopt the same notation as in [32].

$\mathcal{L}_{\mathrm{NP}}^{0}$ reads:

$$
\begin{aligned}
\mathcal{L}_{\mathrm{NP}}^{0}= & \frac{1}{\Lambda^{2}}\left[\left(C_{1}-C_{3}\right)\left(\bar{e}_{L} \gamma^{\mu} \lambda_{e} e_{L}\right)\left(\bar{u}_{L} \gamma_{\mu} \lambda_{u} u_{L}\right)+\left(C_{1}+C_{3}\right)\left(\bar{e}_{L} \gamma^{\mu} \lambda_{e} e_{L}\right)\left(\bar{d}_{L} \gamma_{\mu} \lambda_{d} d_{L}\right)\right. \\
& +\left(C_{1}+C_{3}\right)\left(\bar{\nu}_{L} \gamma^{\mu} \lambda_{e} \nu_{L}\right)\left(\bar{u}_{L} \gamma_{\mu} \lambda_{d} u_{L}\right)+\left(C_{1}-C_{3}\right)\left(\bar{\nu}_{L} \gamma^{\mu} \lambda_{e} \nu_{L}\right)\left(\bar{d}_{L} \gamma_{\mu} \lambda_{d} d_{L}\right) \\
& +\left(2 C_{3}\left(\bar{e}_{L} \gamma^{\mu} \lambda_{e} \nu_{L}\right)\left(\bar{u}_{L} \gamma_{\mu} \lambda_{u d} d_{L}\right)+\text { h.c. }\right)+C_{5}\left(\bar{e}_{R} \gamma^{\mu} \Gamma_{e} e_{R}\right)\left(\bar{d}_{R} \gamma^{\mu} \Gamma_{d} d_{R}\right) \\
& +C_{4}\left(\bar{\nu}_{L} \gamma^{\mu} \lambda_{e} \nu_{L}\right)\left(\bar{d}_{R} \gamma^{\mu} \Gamma_{d} d_{R}\right)+C_{4}\left(\bar{e}_{L} \gamma^{\mu} \lambda_{e} e_{L}\right)\left(\bar{d}_{R} \gamma^{\mu} \Gamma_{d} d_{R}\right) \\
& \left.+C_{6}\left(\bar{u}_{L} \gamma^{\mu} \lambda_{u} u_{L}\right)\left(\bar{e}_{R} \gamma_{\mu} \Gamma_{e} e_{R}\right)+C_{6}\left(\bar{d}_{L} \gamma^{\mu} \lambda_{d} d_{L}\right)\left(\bar{e}_{R} \gamma_{\mu} \Gamma_{e} e_{R}\right)\right] .
\end{aligned}
$$

From this expression we can read the independent parameters of our setup, namely the five Wilson coefficients $C_{i}$ and the matrices $\lambda^{e}, \lambda^{d}, \Gamma^{e}$ and $\Gamma^{d}$.

Following the same steps of refs. [23, 24], we include RGE electroweak effects in leading logarithmic approximation. The operators involved in the running from $\Lambda$ to the EW scale are displayed in table 1 . We find that the effective Lagrangian at the scale $m_{\mathrm{EW}}<\mu<\Lambda$ is given by $\mathcal{L}=\mathcal{L}_{\mathrm{SM}}+\mathcal{L}_{\mathrm{NP}}^{0}+\mathcal{L}_{\text {eff }}$, where $\mathcal{L}_{\text {eff }}$ describes the contribution induced by RGE and can be written as

$$
\mathcal{L}_{\text {eff }}=\delta \mathcal{L}_{\mathrm{SL}}+\delta \mathcal{L}_{\mathrm{L}}+\delta \mathcal{L}_{\mathrm{V}}+\delta \mathcal{L}_{\mathrm{H}}
$$


Explicitly we have

$$
\begin{aligned}
& \delta \mathcal{L}_{\mathrm{SL}}=\frac{L}{16 \pi^{2} \Lambda^{2}}\left\{\left(g_{1}^{2} C_{1}-9 g_{2}^{2} C_{3}\right)\left[Q_{\ell q}^{(1)}\right]_{3333}-\frac{2}{9} g_{1}^{2}\left(C_{1}-C_{4}\right)\left[Q_{\ell q}^{(1)}\right]_{33 s s}\right. \\
& -\frac{2}{3} g_{1}^{2}\left(C_{1}+C_{6}\right)\left[Q_{\ell q}^{(1)}\right]_{s s 33}-\frac{1}{2} C_{1}\left(\left[Y_{u}^{\dagger} Y_{u}\right]_{s 3} \delta_{3 t}+\delta_{s 3}\left[Y_{u}^{\dagger} Y_{u}\right]_{3 t}\right)\left[Q_{\ell q}^{(1)}\right]_{33 s t} \\
& +\left(-3 g_{2}^{2} C_{1}+C_{3}\left(6 g_{2}^{2}+g_{1}^{2}\right)\right)\left[Q_{\ell q}^{(3)}\right]_{3333}-2 g_{2}^{2} C_{3}\left[Q_{\ell q}^{(3)}\right]_{33 s s}-\frac{2}{3} g_{2}^{2} C_{3}\left[Q_{\ell q}^{(3)}\right]_{s s 33} \\
& -\frac{1}{2} C_{3}\left(\left[Y_{u}^{\dagger} Y_{u}\right]_{s 3} \delta_{3 t}+\delta_{s 3}\left[Y_{u}^{\dagger} Y_{u}\right]_{3 t}\right)\left[Q_{\ell q}^{(3)}\right]_{33 s t}-\frac{8}{9} g_{1}^{2}\left(C_{1}-C_{4}\right)\left[Q_{\ell u}\right]_{33 s s} \\
& +2\left[Y_{u}\right]_{s 3}\left[Y_{u}^{\dagger}\right]_{3 t} C_{1}\left[Q_{\ell u}\right]_{33 s t}+2 g_{1}^{2} C_{4}\left[Q_{\ell d}\right]_{3333}+\frac{4}{9} g_{1}^{2}\left(C_{1}-C_{4}\right)\left[Q_{\ell d}\right]_{33 s s} \\
& -\frac{2}{3} g_{1}^{2}\left(C_{4}+C_{5}\right)\left[Q_{\ell d}\right]_{s s 33}-2 g_{1}^{2} C_{6}\left[Q_{q e}\right]_{3333}-\frac{4}{3} g_{1}^{2}\left(C_{1}+C_{6}\right)\left[Q_{q e}\right]_{33 s s} \\
& -\frac{1}{2} C_{6}\left(\left[Y_{u}^{\dagger} Y_{u}\right]_{s 3} \delta_{3 t}+\delta_{s 3}\left[Y_{u}^{\dagger} Y_{u}\right]_{3 t}\right)\left[Q_{q e}\right]_{s t 33}+\frac{8}{9} g_{1}^{2}\left(C_{5}-C_{6}\right)\left[Q_{e u}\right]_{33 s s} \\
& +2\left[Y_{u}\right]_{s 3}\left[Y_{u}^{\dagger}\right]_{3 t} C_{6}\left[Q_{e u}\right]_{33 s t}-4 g_{1}^{2} C_{5}\left[Q_{e d}\right]_{3333}+\frac{2}{9} g_{1}^{2}\left(C_{5}-C_{6}\right)\left[Q_{q e}\right]_{33 s s} \\
& \left.-\frac{4}{9} g_{1}^{2}\left(C_{5}-C_{6}\right)\left[Q_{e d}\right]_{33 s s}-\frac{4}{3} g_{1}^{2}\left(C_{4}+C_{5}\right)\left[Q_{e d}\right]_{s s 33}\right\}, \\
& \delta \mathcal{L}_{\mathrm{L}}=\frac{L}{16 \pi^{2} \Lambda^{2}}\left\{\left(\frac{2}{3} g_{1}^{2}\left(C_{1}-C_{4}\right)+2 g_{2}^{2} C_{3}\right)\left[Q_{\ell \ell}\right]_{33 s s}-4 g_{2}^{2} C_{3}\left[Q_{\ell \ell}\right]_{3 s s 3}\right. \\
& \left.+\frac{4}{3} g_{1}^{2}\left(C_{1}-C_{4}\right)\left[Q_{\ell e}\right]_{33 s s}-\frac{2}{3} g_{1}^{2}\left(C_{5}-C_{6}\right)\left[Q_{\ell e}\right]_{s s 33}\right\}, \\
& \delta \mathcal{L}_{\mathrm{V}}=\frac{L}{16 \pi^{2} \Lambda^{2}}\left\{\left(-6 C_{1} \lambda_{33}^{u} y_{t}^{2}-\frac{2}{3} g_{1}^{2}\left(C_{1}-C_{4}\right)\right)\left[Q_{H \ell}^{(1)}\right]_{33}\right. \\
& +\left(6 C_{3} \lambda_{33}^{u} y_{t}^{2}-2 g_{1}^{2}\left(C_{1}-C_{4}\right)\right)\left[Q_{H \ell}^{(3)}\right]_{33}+\frac{2}{3} g_{1}^{2}\left(C_{1}+C_{6}\right)\left[Q_{H q}^{(1)}\right]_{33} \\
& -\frac{2}{3} g_{2}^{2} C_{3}\left[Q_{H q}^{(3)}\right]_{33}+\left(\frac{2}{3} g_{1}^{2}\left(C_{5}-C_{6}\right)-6 C_{6} \lambda_{33}^{u} y_{t}^{2}\right)\left[Q_{H e}\right]_{33} \\
& \left.+\frac{2}{3} g_{1}^{2}\left(C_{4}+C_{5}\right)\left[Q_{H d}\right]_{33}\right\} \\
& \delta \mathcal{L}_{\mathrm{H}}=\frac{L}{16 \pi^{2} \Lambda^{2}}\left\{\frac{2}{9} g_{1}^{2}\left(C_{1}+C_{6}\right)\left[Q_{q q}^{(1)}\right]_{33 s s}-\frac{2}{3} g_{2}^{2} C_{3}\left[Q_{q q}^{(3)}\right]_{33 s s}+\frac{8}{9} g_{1}^{2}\left(C_{1}+C_{6}\right)\left[Q_{q u}^{(1)}\right]_{33 s s}\right. \\
& \left.-\frac{4}{9} g_{1}^{2}\left(C_{1}+C_{6}\right)\left[Q_{q d}^{(1)}\right]_{33 s s}+\frac{2}{9} g_{1}^{2}\left(C_{4}+C_{5}\right)\left[Q_{q u}^{(1)}\right]_{s s 33}-\frac{4}{9} g_{1}^{2}\left(C_{4}+C_{5}\right)\left[Q_{d d}\right]_{33 s s}\right\} .
\end{aligned}
$$

where $L=\log \frac{\Lambda}{\mu}$, the sum over repeated flavour indices is understood and the results are expressed in the interaction basis. In the above expressions, we have sistematically included both gauge and top yukawa interactions, exploiting the results of [33, 34]. ${ }^{2}$ Instead, we have neglected down-quark and leptons yukawas since their effects are very small.

\footnotetext{
${ }^{2}$ Notice that QCD interactions do not renormalise the quark currents $V \pm A$ analysed here.
} 
After the breaking of the electroweak symmetry, $\delta \mathcal{L}_{\mathrm{V}}$ induces modifications of the $W$ and $Z$ couplings to fermions. The full $Z$ and $W$ Lagrangian reads:

$$
\mathcal{L}_{\mathrm{Z}, \mathrm{W}}=-\frac{g_{2}}{c_{\mathrm{W}}} Z_{\mu} J^{\mu 0}-\frac{g_{2}}{\sqrt{2}}\left(W_{\mu}^{+} J^{\mu,-}+\text { h.c. }\right)
$$

where

$$
\begin{aligned}
J^{\mu, 0} & =\sum_{f}\left[\left(g_{L, \mathrm{SM}}^{f}+\Delta g_{L}^{f}\right)_{i j} \bar{f}_{i L} \gamma^{\mu} f_{j L}+\left(g_{R, \mathrm{SM}}^{f}+\Delta g_{R}^{f}\right)_{i j} \bar{f}_{i R} \gamma^{\mu} f_{j R}\right] \\
J^{\mu,-} & =\left(g_{\mathrm{SM}}^{\ell}+\Delta g^{\ell}\right)_{i j} \bar{\nu}_{i L} \gamma^{\mu} e_{j L}+\left(g_{\mathrm{SM}}^{q}+\Delta g^{q}\right)_{i j} \bar{u}_{i L} \gamma^{\mu} d_{j L},
\end{aligned}
$$

and $c_{\mathrm{W}}=\cos \theta_{\mathrm{W}}$. These expressions include the SM contribution

$$
\begin{aligned}
\left(g_{L, \mathrm{SM}}^{f}\right)_{i j} & =g_{L, \mathrm{SM}}^{f} \delta_{i j}=\left(T_{3}^{f}-q_{f} s_{\mathrm{W}}^{2}\right) \delta_{i j} \\
\left(g_{R, \mathrm{SM}}^{f}\right)_{i j} & =g_{R, \mathrm{SM}}^{f} \delta_{i j}=-q_{f} s_{\mathrm{W}}^{2} \delta_{i j} \\
\left(g_{\mathrm{SM}}^{\ell}\right)_{i j} & =\delta_{i j} \\
\left(g_{\mathrm{SM}}^{q}\right)_{i j} & =\left(V_{\mathrm{CKM}}\right)_{i j}
\end{aligned}
$$

and the NP contribution, encoded in the deviations $\Delta g_{L, R}^{f}$ and $\Delta g^{q / \ell}$. For the $Z$ couplings we have

$$
\begin{aligned}
& \left(\Delta g_{L}^{\nu}\right)_{i j}=\frac{v^{2}}{\Lambda^{2}} \frac{L}{16 \pi^{2}}\left[\frac{g_{1}^{2}}{3}\left(C_{1}-C_{4}\right)-g_{2}^{2} C_{3}+3 \lambda_{33}^{u} y_{t}^{2}\left(C_{1}+C_{3}\right)\right] \lambda_{i j}^{e} \\
& \left(\Delta g_{L}^{e}\right)_{i j}=\frac{v^{2}}{\Lambda^{2}} \frac{L}{16 \pi^{2}}\left[\frac{g_{1}^{2}}{3}\left(C_{1}-C_{4}\right)+g_{2}^{2} C_{3}+3 \lambda_{33}^{u} y_{t}^{2}\left(C_{1}-C_{3}\right)\right] \lambda_{i j}^{e} \\
& \left(\Delta g_{L}^{u}\right)_{i j}=\frac{v^{2}}{\Lambda^{2}} \frac{L}{16 \pi^{2}} \frac{1}{3}\left[-g_{2}^{2} C_{3}-g_{1}^{2}\left(C_{1}+C_{6}\right)\right] \lambda_{i j}^{u} \\
& \left(\Delta g_{L}^{d}\right)_{i j}=\frac{v^{2}}{\Lambda^{2}} \frac{L}{16 \pi^{2}} \frac{1}{3}\left[g_{2}^{2} C_{3}-g_{1}^{2}\left(C_{1}+C_{6}\right)\right] \lambda_{i j}^{d} \\
& \left(\Delta g_{R}^{e}\right)_{i j}=\frac{v^{2}}{\Lambda^{2}} \frac{L}{16 \pi^{2}}\left[-\frac{1}{3} g_{1}^{2}\left(C_{5}-C_{6}\right)+3 C_{6} \lambda_{33}^{u} y_{t}^{2}\right] \Gamma_{i j}^{e} \\
& \left(\Delta g_{R}^{u}\right)_{i j}=0 \\
& \left(\Delta g_{R}^{d}\right)_{i j}=\frac{v^{2}}{\Lambda^{2}} \frac{L}{16 \pi^{2}}\left[-\frac{1}{3} g_{1}^{2}\left(C_{4}+C_{5}\right)\right] \Gamma_{i j}^{d},
\end{aligned}
$$

while for $W$ couplings we find

$$
\begin{aligned}
& \left(\Delta g^{\ell}\right)_{i j}=\frac{v^{2}}{\Lambda^{2}} \frac{L}{16 \pi^{2}}\left[6 C_{3} \lambda_{33}^{u} y_{t}^{2}-2 g_{2}^{2} C_{3}\right] \lambda_{i j}^{e} \\
& \left(\Delta g^{q}\right)_{i j}=\frac{v^{2}}{\Lambda^{2}} \frac{L}{16 \pi^{2}}\left[-\frac{2}{3} g_{2}^{2} C_{3}\right] \lambda_{i j}^{u d} .
\end{aligned}
$$

We see that RGE effects induce flavour and flavour universality violating interactions, which are absent in the SM. We have explicitly checked that the dependence on the unphysical scale $\mu$ cancels when physical quantities are computed. For $W$ and $Z$ decays, 


\begin{tabular}{|c|l|}
\hline$Q_{i}$ & $\xi_{i}$ \\
\hline$\left(\bar{\nu}_{i L} \gamma_{\mu} \nu_{j L}\right)\left(\bar{\nu}_{k L} \gamma_{\mu} \nu_{n L}\right)$ & $\lambda_{i j}^{e} \delta_{k n}\left[-6 y_{t}^{2} \lambda_{33}^{u}\left(C_{1}+C_{3}\right)\right]$ \\
\hline$\left(\bar{\nu}_{i L} \gamma_{\mu} \nu_{j L}\right)\left(\bar{e}_{k L} \gamma_{\mu} e_{n L}\right)$ & $\begin{array}{l}\lambda_{i j}^{e} \delta_{k n}\left[\frac{4}{3} e^{2}\left(C_{1}+3 C_{3}-C_{4}\right)-12\left(-\frac{1}{2}+s_{\mathrm{W}}^{2}\right) y_{t}^{2} \lambda_{33}^{u}\left(C_{1}+C_{3}\right)\right] \\
+\delta_{i j} \lambda_{k n}^{e}\left[-6 y_{t}^{2} \lambda_{33}^{u}\left(C_{1}-C_{3}\right)\right]\end{array}$ \\
\hline$\left(\bar{\nu}_{i L} \gamma^{\mu} \nu_{j L}\right)\left(\bar{e}_{k R} \gamma_{\mu} e_{n R}\right)$ & $\begin{array}{l}\left.\lambda_{i j}^{e} \delta_{k n}\left[\frac{4}{3} e^{2}\left(C_{1}+3 C_{3}-C_{4}\right)-12 s_{\mathrm{w}}^{2} y_{t}^{2} \lambda_{33}^{u}\left(C_{1}+C_{3}\right)\right)\right] \\
+\delta_{i j} \Gamma_{k n}^{e}\left[-6 C_{6} \lambda_{33}^{u} y_{t}^{2}\right]\end{array}$ \\
\hline$\left(\bar{e}_{i L} \gamma^{\mu} e_{j L}\right)\left(\bar{e}_{k L} \gamma_{\mu} e_{n L}\right)$ & $\delta_{i j} \lambda_{k n}^{e}\left[\frac{4}{3} e^{2}\left(C_{1}-3 C_{3}-C_{4}\right)-12\left(-\frac{1}{2}+s_{\mathrm{W}}^{2}\right) y_{t}^{2} \lambda_{33}^{u}\left(C_{1}-C_{3}\right)\right]$ \\
\hline$\left(\bar{e}_{i L} \gamma^{\mu} e_{j L}\right)\left(\bar{e}_{k R} \gamma_{\mu} e_{n R}\right)$ & $\lambda_{i j}^{e} \delta_{k n}\left[\frac{4}{3} e^{2}\left(C_{1}-3 C_{3}-C_{4}\right)-12 s_{\mathrm{w}}^{2} y_{t}^{2} \lambda_{33}^{u}\left(C_{1}-C_{3}\right)\right]$ \\
& $+\delta_{i j} \Gamma_{k n}^{e}\left[-\frac{4}{3} e^{2}\left(C_{5}-C_{6}\right)-12\left(-\frac{1}{2}+s_{\mathrm{W}}^{2}\right) C_{6} \lambda_{33}^{u} y_{t}^{2}\right]$ \\
\hline$\left(\bar{e}_{i R} \gamma^{\mu} e_{j R}\right)\left(\bar{e}_{k R} \gamma_{\mu} e_{n R}\right)$ & $\delta_{i j} \Gamma_{k n}^{e}\left[-\frac{4}{3} e^{2}\left(C_{5}-C_{6}\right)-12 s_{\mathrm{W}}^{2} C_{6} \lambda_{33}^{u} y_{t}^{2}\right]$ \\
\hline$\left(\bar{\nu}_{i L} \gamma_{\mu} e_{j L}\right)\left(\bar{e}_{k L} \gamma_{\mu} \nu_{n L}\right)$ & $\left(\lambda_{i j}^{e} \delta_{k n}+\delta_{i j} \lambda_{k n}^{e}\right)\left[-12 y_{t}^{2} \lambda_{33}^{u} C_{3}\right]$ \\
\hline
\end{tabular}

Table 2. Operators $Q_{i}$ and coefficients $\xi_{i}$ for the purely leptonic part of the effective Lagrangian $\mathcal{L}_{\text {eff }}^{\mathrm{EW}}$. We set $\sin ^{2} \theta_{W} \equiv s_{\mathrm{w}}^{2}$.

this approximately amounts to make use of $\mathcal{L}_{Z, \mathrm{~W}}$ in eq. (2.11) in the tree-level approximation by replacing $\mu$ with the electroweak scale.

At the scale $\mu=m_{\mathrm{EW}}$ we match the effective Lagrangian $\mathcal{L}_{\text {eff }}$ with a new Lagrangian $\mathcal{L}_{\text {eff }}^{\text {EW }}$ obtained by integrating out the $W, Z$ bosons and the top quark. For the vector bosons $W$ and $Z$ we work at the tree-level. Disregarding the purely hadronic contribution, we get:

$$
\mathcal{L}_{\mathrm{eff}}^{\mathrm{EW}}=\frac{1}{\Lambda^{2}} \sum_{i} C_{i}\left(m_{\mathrm{EW}}\right) Q_{i}=\frac{1}{16 \pi^{2} \Lambda^{2}} \log \frac{\Lambda}{m_{\mathrm{EW}}} \sum_{i} \xi_{i} Q_{i}
$$

The operators $Q_{i}$ and their coefficients $\xi_{i}$ are listed in the tables 2, 3, 4 and 5 .

Below the electroweak scale only the residual electromagnetic gauge symmetry is relevant to our discussion, and the effective theory consists of a combination of $\mathrm{U}(1)_{\mathrm{em}}$-invariant operators whose Wilson coefficients run under the effect of QED interactions only. By lowering the scale $\mu$ we first cross the bottom quark mass threshold, then the charm one. When crossing a threshold we integrate out the corresponding quark and match the theory to a new one. At the scale $\mu \approx 1 \mathrm{GeV}$ we get the following result for the effective Lagrangian $\mathcal{L}_{\text {eff }}^{\mathrm{QED}}$ :

$$
\begin{aligned}
\mathcal{L}_{\mathrm{eff}}^{\mathrm{QED}} & =\frac{1}{\Lambda^{2}} \sum_{i} \mathcal{C}_{i}\left(m_{\mathrm{EW}}\right) Q_{i}+\frac{1}{\Lambda^{2}} \sum_{i} \delta \mathcal{C}_{i}(\mu) Q_{i}^{\mathrm{em}} \\
& =\frac{1}{16 \pi^{2} \Lambda^{2}} \log \frac{\Lambda}{m_{\mathrm{EW}}} \sum_{i} \xi_{i} Q_{i}+\frac{1}{16 \pi^{2} \Lambda^{2}} \log \frac{m_{\mathrm{EW}}}{\mu} \sum_{i} \delta \xi_{i} Q_{i}^{\mathrm{em}}
\end{aligned}
$$

where the $\mathrm{U}(1)_{\mathrm{em}}$-invariant operators $Q_{i}^{\mathrm{em}}$ and their coefficients $\delta \xi_{i}$ are collected in tables 6 , 7 and 8 . 


\begin{tabular}{|c|c|}
\hline$Q_{i}$ & $\xi_{i}$ \\
\hline$\left(\bar{\nu}_{i L} \gamma_{\mu} \nu_{j L}\right)\left(\bar{u}_{k L} \gamma^{\mu} u_{n L}\right)$ & $\lambda_{i j}^{e} \lambda_{k n}^{u}\left[\left(g_{1}^{2}-3 g_{2}^{2}\right)\left(C_{1}+C_{3}\right)\right]$ \\
& $+\lambda_{i j}^{e} \delta_{k n}\left[-\frac{8}{9} e^{2}\left(C_{1}+3 C_{3}-C_{4}\right)-12\left(\frac{1}{2}-\frac{2}{3} s_{\mathrm{W}}^{2}\right) y_{t}^{2} \lambda_{33}^{u}\left(C_{1}+C_{3}\right)\right]$ \\
& $+\lambda_{i j}^{e}\left(\lambda_{k 3}^{u} \delta_{3 n}+\delta_{k 3} \lambda_{3 n}^{u}\right)\left[-\frac{1}{2} y_{t}^{2}\left(C_{1}+C_{3}\right)\right]$ \\
\hline$\left(\bar{\nu}_{i L} \gamma_{\mu} \nu_{j L}\right)\left(\bar{u}_{k R} \gamma^{\mu} u_{n R}\right)$ & $\lambda_{i j}^{e} \delta_{k n}\left[-\frac{8}{9} e^{2}\left(C_{1}+3 C_{3}-C_{4}\right)+8 s_{\mathrm{w}}^{2} y_{t}^{2} \lambda_{33}^{u}\left(C_{1}+C_{3}\right)\right]$ \\
& $+\lambda_{i j}^{e} \delta_{k 3} \delta_{3 n}\left[2 y_{t}^{2} \lambda_{33}^{u} C_{1}\right]$
\end{tabular}

Table 3. Operators $Q_{i}$ and coefficients $\xi_{i}$ for the semileptonic part of the effective Lagrangian $\mathcal{L}_{\text {eff }}^{\text {EW }}$ involving neutrinos and neutral currents. Generation indices run from 1 to 3, exception made for up-type quarks where $k, n=1,2$. We set $\sin ^{2} \theta_{W} \equiv s_{\mathrm{W}}^{2}$.

\section{Observables}

This section addresses the phenomenological consequences of Lagrangian (2.2), making use of the RGE-improved low-energy effective field theory (EFT) derived in the previous section. The NP contribution to the observables is parametrised in terms of the free parameters of $\mathcal{L}_{\mathrm{NP}}^{0}$, namely the five $C_{i}$ and the matrices $\lambda^{e}, \lambda^{d}, \Gamma^{e}$ and $\Gamma^{d}$. In order to simplify our phenomenological analysis, we assume real entries in $\lambda^{e / d}$ and $\Gamma^{e / d}$, negligible mixing with the first generation in the matrices $\lambda^{e / d}$ and $\Gamma^{e / d}, \lambda_{1 i}^{e / d}=\Gamma_{1 i}^{e / d}=0(i=1,2,3)$ and a small mixing approximation, ${ }^{3}$ implying

$$
\lambda_{22}^{e / d} \approx\left|\lambda_{23}^{e / d}\right|^{2} \ll \lambda_{33}^{e / d} \quad \lambda_{33}^{e / d} \approx 1
$$

and similarly for $\Gamma^{e / d}$. As a result, the parameters involved in our analysis are $C_{1}, C_{3}$, $C_{4}, C_{5}, C_{6}, \lambda_{23}^{e / d}, \Gamma_{23}^{e / d}$. Beyond semileptonic B-decays, we focus on fully leptonic processes and leptonic decays of the $Z$ vector boson as they are the only processes that compete with semileptonic B-decays in constraining our NP parameter space. The structure of this section is as follows. In section 3.1, we discuss how to address both charged- and neutralcurrent $B$ anomalies within our framework. In section 3.2, we discuss the most relevant tree-level phenomenology connected with the $B$ anomalies. In section 3.3 , we proceed to study observables in the leptonic sector receiving large contributions at loop-level. In section 3.4, we first perform a global numerical analysis in a phenomenologically relevant scenario, where NP affects dominantly the Wilson coefficient $\mathcal{C}^{9}$. As a further step, we extend the analysis by exploring the available parameter space in full generality.

\footnotetext{
${ }^{3}$ The largest mixing arises from $\lambda_{23}^{e} \approx 0.3$. In our numerical analysis we will let $\left|\lambda_{23}^{e}\right|$ and $\left|\Gamma_{23}^{e}\right|$ vary up to 0.5 by using complete formulae.
} 


\begin{tabular}{|c|c|}
\hline$Q_{i}$ & $\xi_{i}$ \\
\hline$\left(\bar{e}_{i L} \gamma_{\mu} e_{j L}\right)\left(\bar{u}_{k L} \gamma^{\mu} u_{n L}\right)$ & $\begin{aligned} & \lambda_{i j}^{e} \lambda_{k n}^{u}\left[\left(g_{1}^{2}+3 g_{2}^{2}\right) C_{1}-\left(g_{1}^{2}+15 g_{2}^{2}\right) C_{3}\right] \\
+ & \lambda_{i j}^{e} \delta_{k n}\left[-\frac{8}{9} e^{2}\left(C_{1}-3 C_{3}-C_{4}\right)-12\left(\frac{1}{2}-\frac{3}{2} s_{\mathrm{w}}^{2}\right) y_{t}^{2} \lambda_{33}^{u}\left(C_{1}-C_{3}\right)\right] \\
+ & \delta_{i j} \lambda_{k n}^{u}\left[-\frac{4}{3} e^{2}\left(C_{1}-C_{3}+C_{6}\right)\right] \\
+ & \lambda_{i j}^{e}\left(\lambda_{k 3}^{u} \delta_{3 n}+\delta_{k 3} \lambda_{3 n}^{u}\right)\left[-\frac{1}{2} y_{t}^{2}\left(C_{1}-C_{3}\right)\right]\end{aligned}$ \\
\hline$\left(\bar{e}_{i L} \gamma_{\mu} e_{j L}\right)\left(\bar{u}_{k R} \gamma^{\mu} u_{n R}\right)$ & $\begin{aligned} & \lambda_{i j}^{e} \delta_{k n}\left[-\frac{8}{9} e^{2}\left(C_{1}-3 C_{3}-C_{4}\right)+8 s_{\mathrm{w}}^{2} y_{t}^{2} \lambda_{33}^{u}\left(C_{1}-C_{3}\right)\right] \\
+ & \lambda_{i j}^{e} \delta_{k 3} \delta_{3 n}\left[2 y_{t}^{2} \lambda_{33}^{u} C_{1}\right]\end{aligned}$ \\
\hline$\left(\bar{e}_{i R} \gamma_{\mu} e_{j R}\right)\left(\bar{u}_{k L} \gamma^{\mu} u_{n L}\right)$ & $\begin{aligned} & \Gamma_{i j}^{e} \lambda_{k n}^{u}\left[-2 g_{1}^{2} C_{6}\right] \\
+ & \Gamma_{i j}^{e} \delta_{k n}\left[\frac{8}{9} e^{2}\left(C_{5}-C_{6}\right)-12\left(\frac{1}{2}-\frac{2}{3} s_{\mathrm{w}}^{2}\right) y_{t}^{2} \lambda_{33}^{u} C_{6}\right] \\
+ & \delta_{i j} \lambda_{k n}^{u}\left[-\frac{4}{3} e^{2}\left(C_{1}-C_{3}+C_{6}\right)\right] \\
+ & \Gamma_{i j}^{e}\left(\lambda_{k 3}^{u} \delta_{3 n}+\delta_{k 3} \lambda_{3 n}^{u}\right)\left[-\frac{1}{2} y_{t}^{2} C_{6}\right]\end{aligned}$ \\
\hline$\left(\bar{e}_{i R} \gamma_{\mu} e_{j R}\right)\left(\bar{u}_{k R} \gamma^{\mu} u_{n R}\right)$ & $\begin{aligned} & \Gamma_{i j}^{e} \delta_{k n}\left[\frac{8}{9} e^{2}\left(C_{5}-C_{6}\right)+8 s_{\mathrm{w}}^{2} y_{t}^{2} \lambda_{33}^{u} C_{6}\right] \\
+ & \Gamma_{i j}^{e} \delta_{3 k} \delta_{3 n}\left[2 y_{t}^{2} \lambda_{33}^{u} C_{6}\right]\end{aligned}$ \\
\hline$\left(\bar{e}_{i L} \gamma_{\mu} e_{j L}\right)\left(\bar{d}_{k L} \gamma^{\mu} d_{n L}\right)$ & $\begin{aligned} & \lambda_{i j}^{e} \lambda_{k n}^{d}\left[\left(g_{1}^{2}-3 g_{2}^{2}\right)\left(C_{1}+C_{3}\right)\right] \\
+ & \lambda_{i j}^{e} \delta_{k n}\left[\frac{4}{9} e^{2}\left(C_{1}-3 C_{3}-C_{4}\right)-12\left(-\frac{1}{2}+\frac{1}{3} s_{\mathrm{w}}^{2}\right) y_{t}^{2} \lambda_{33}^{u}\left(C_{1}-C_{3}\right)\right] \\
+ & \delta_{i j} \lambda_{k n}^{d}\left[-\frac{4}{3} e^{2}\left(C_{1}+C_{3}+C_{6}\right)\right] \\
+ & \lambda_{i j}^{e}\left(\left(\lambda^{u d \dagger}\right)_{k 3} V_{3 n}^{\mathrm{CKM}}+\left(V^{\mathrm{CKM} \dagger}\right)_{k 3} \lambda_{3 n}^{u d}\right)\left[-\frac{1}{2} y_{t}^{2}\left(C_{1}+C_{3}\right)\right]\end{aligned}$ \\
\hline$\left(\bar{e}_{i L} \gamma_{\mu} e_{j L}\right)\left(\bar{d}_{k R} \gamma^{\mu} d_{n R}\right)$ & $\begin{aligned} & \lambda_{i j}^{e} \Gamma_{k n}^{d}\left[2 g_{1}^{2} C_{4}\right] \\
+ & \lambda_{i j}^{e} \delta_{k n}\left[\frac{4}{9} e^{2}\left(C_{1}-3 C_{3}-C_{4}\right)-4 s_{\mathrm{W}}^{2} y_{t}^{2} \lambda_{33}^{u}\left(C_{1}-C_{3}\right)\right] \\
+ & \delta_{i j} \Gamma_{k n}^{d}\left[-\frac{4}{3} e^{2}\left(C_{4}+C_{5}\right)\right]\end{aligned}$ \\
\hline$\left(\bar{e}_{i R} \gamma_{\mu} e_{j R}\right)\left(\bar{d}_{k L} \gamma^{\mu} d_{n L}\right)$ & $\begin{aligned} & \Gamma_{i j}^{e} \lambda_{k n}^{d}\left[-2 g_{1}^{2} C_{6}\right] \\
+ & \Gamma_{i j}^{e} \delta_{k n}\left[-\frac{4}{9} e^{2}\left(C_{5}-C_{6}\right)-12\left(-\frac{1}{2}+\frac{1}{3} s_{\mathrm{W}}^{2}\right) y_{t}^{2} \lambda_{33}^{u} C_{6}\right] \\
+ & \delta_{i j} \lambda_{k n}^{d}\left[-\frac{4}{3} e^{2}\left(C_{1}+C_{3}+C_{6}\right)\right] \\
+ & \Gamma_{i j}^{e}\left(\left(\lambda^{u d \dagger}\right)_{k 3} V_{3 n}^{\mathrm{CKM}}+\left(V^{\mathrm{CKM} \dagger}\right)_{k 3} \lambda_{3 n}^{u d}\right)\left[-\frac{1}{2} y_{t}^{2} C_{6}\right]\end{aligned}$ \\
\hline$\left(\bar{e}_{i R} \gamma_{\mu} e_{j R}\right)\left(\bar{d}_{k R} \gamma^{\mu} d_{n R}\right)$ & $\begin{aligned} & \Gamma_{i j}^{e} \Gamma_{k n}^{d}\left[-4 g_{1}^{2} C_{5}\right] \\
+ & \Gamma_{i j}^{e} \delta_{k n}\left[-\frac{4}{9} e^{2}\left(C_{5}-C_{6}\right)-4 s_{\mathrm{w}}^{2} y_{t}^{2} \lambda_{33}^{u} C_{6}\right] \\
+ & \delta_{i j} \Gamma_{k n}^{d}\left[-\frac{4}{3} e^{2}\left(C_{4}+C_{5}\right]\right.\end{aligned}$ \\
\hline
\end{tabular}

Table 4. Operators $Q_{i}$ and coefficients $\xi_{i}$ for the semileptonic part of the effective Lagrangian $\mathcal{L}_{\text {eff }}^{\text {EW }}$ involving charged leptons and neutral currents. Generation indices run from 1 to 3, exception made for up-type quarks where $k, n=1,2$. We set $\sin ^{2} \theta_{W} \equiv s_{\mathrm{W}}^{2}$. 


\begin{tabular}{|c|c|}
\hline$Q_{i}$ & $\xi_{i}$ \\
\hline$\left(\bar{e}_{i L} \gamma_{\mu} \nu_{j L}\right)\left(\bar{u}_{k L} \gamma^{\mu} d_{n L}\right)$ & $\lambda_{i j}^{e} \lambda_{k n}^{u d}\left[-6 g_{2}^{2} C_{1}+2\left(6 g_{2}^{2}+g_{1}^{2}\right) C_{3}\right]$ \\
& $+\lambda_{i j}^{e} V_{k n}^{\mathrm{CKM}}\left[-12 y_{t}^{2} \lambda_{33}^{u} C_{3}\right]$ \\
& $+\lambda_{i j}^{e}\left(\lambda_{k 3}^{u} V_{3 n}^{\mathrm{CKM}}+\delta_{k 3} \lambda_{3 n}^{u d}\right)\left[-y_{t}^{2} C_{3}\right]$ \\
\hline
\end{tabular}

Table 5. Operators $Q_{i}$ and coefficients $\xi_{i}$ for the semileptonic part of the effective Lagrangian $\mathcal{L}_{\text {eff }}^{\mathrm{EW}}$ involving charged currents. For up-type quarks the indices run from 1 to 2 . The $\xi_{i}$ coefficient for the Hermitian conjugate operator can be easily derived.

\begin{tabular}{|c|c|}
\hline$Q_{i}^{\mathrm{em}}$ & $\delta \xi_{i}$ \\
\hline$\left(\bar{\nu}_{i L} \gamma_{\mu} \nu_{j L}\right)\left(\bar{\nu}_{k L} \gamma^{\mu} \nu_{n L}\right)$ & 0 \\
\hline$\left(\bar{\nu}_{i L} \gamma_{\mu} \nu_{j L}\right)\left(\bar{e}_{k} \gamma^{\mu} e_{n}\right)$ & $\lambda_{i j}^{e} \delta_{k n} \cdot \frac{4}{3} e^{2}\left[\left(C_{1}+3 C_{3}-C_{4}\right)-2\left(C_{1}+C_{3}\right)\left(\lambda_{33}^{u}+\hat{\lambda}_{22}^{u} \log \frac{m_{c}}{\mu}\right)\right.$ \\
& $\left.+\left(\left(C_{1}-C_{3}\right) \hat{\lambda}_{33}^{d}+C_{4} \hat{\Gamma}_{33}^{d}\right) \log \frac{m_{b}}{\mu}\right]$ \\
\hline$\left(\bar{e}_{i L} \gamma_{\mu} e_{j L}\right)\left(\bar{e}_{k} \gamma^{\mu} e_{n}\right)$ & $\lambda_{i j}^{e} \delta_{k n} \cdot \frac{4}{3} e^{2}\left[\left(C_{1}-3 C_{3}-C_{4}\right)-2\left(C_{1}-C_{3}\right)\left(\lambda_{33}^{u}+\hat{\lambda}_{22}^{u} \log \frac{m_{c}}{\mu}\right)\right.$ \\
& $\left.+\left(\left(C_{1}+C_{3}\right) \hat{\lambda}_{33}^{d}+C_{4} \hat{\Gamma}_{33}^{d}\right) \log \frac{m_{b}}{\mu}\right]$ \\
\hline$\left(\bar{e}_{i R} \gamma_{\mu} e_{j R}\right)\left(\bar{e}_{k} \gamma^{\mu} e_{n}\right)$ & $\Gamma_{i j}^{e} \delta_{k n} \cdot \frac{4}{3} e^{2}\left[\left(C_{6}-C_{5}\right)-2 C_{6}\left(\lambda_{33}^{u}+\hat{\lambda}_{22}^{u} \log \frac{m_{c}}{\mu}\right)\right.$ \\
& $\left.+\left(C_{6} \hat{\lambda}_{33}^{d}+C_{5} \hat{\Gamma}_{33}^{d}\right) \log \frac{m_{b}}{\mu}\right]$ \\
\hline
\end{tabular}

Table 6. Operators $Q_{i}^{\mathrm{em}}$ and coefficients $\delta \xi_{i}$ for the purely leptonic part of the effective Lagrangian $\delta \mathcal{L}_{\text {eff }}^{\mathrm{QED}}$. We set $\hat{\lambda}_{i i}^{u, d}=\lambda_{i i}^{u, d} / \log \frac{m_{\mathrm{EW}}}{\mu}$.

\begin{tabular}{|c|c|}
\hline$Q_{i}^{e m}$ & $\delta \xi_{i}$ \\
\hline$\left(\bar{\nu}_{i L} \gamma_{\mu} \nu_{j L}\right)\left(\bar{u}_{k} \gamma^{\mu} u_{n}\right)$ & $\lambda_{i j}^{e} \delta_{k n}\left(-\frac{8}{9} e^{2}\right)\left[\left(C_{1}+3 C_{3}-C_{4}\right)-2\left(C_{1}+C_{3}\right)\left(\lambda_{33}^{u}+\hat{\lambda}_{22}^{u} \log \frac{m_{c}}{\mu}\right)\right.$ \\
& $\left.+\left(\left(C_{1}-C_{3}\right) \hat{\lambda}_{33}^{d}+C_{4} \hat{\Gamma}_{33}^{d}\right) \log \frac{m_{b}}{\mu}\right]$ \\
\hline$\left(\bar{\nu}_{i L} \gamma_{\mu} \nu_{j L}\right)\left(\bar{d}_{k} \gamma^{\mu} d_{n}\right)$ & $\lambda_{i j}^{e} \delta_{k n} \cdot \frac{4}{9} e^{2}\left[\left(C_{1}+3 C_{3}-C_{4}\right)-2\left(C_{1}+C_{3}\right)\left(\lambda_{33}^{u}+\hat{\lambda}_{22}^{u} \log \frac{m_{c}}{\mu}\right)\right.$ \\
& $\left.+\left(\left(C_{1}-C_{3}\right) \hat{\lambda}_{33}^{d}+C_{4} \hat{\Gamma}_{33}^{d}\right) \log \frac{m_{b}}{\mu}\right]$ \\
\hline
\end{tabular}

Table 7. Operators $Q_{i}^{\mathrm{em}}$ and coefficients $\delta \xi_{i}$ for the semileptonic part of the effective Lagrangian $\delta \mathcal{L}_{\text {eff }}^{\mathrm{QED}}$ involving neutrinos and neutral currents. For the down-type quarks generation indices run from 1 to 2 ,while for up-type quarks we only keep the first generation. We set $\hat{\lambda}_{i i}^{u, d}=\lambda_{i i}^{u, d} / \log \frac{m_{E W}}{\mu}$. 


\begin{tabular}{|c|c|}
\hline$Q_{i}^{e m}$ & $\delta \xi_{i}$ \\
\hline$\left(\bar{e}_{i L} \gamma_{\mu} e_{j L}\right)\left(\bar{u}_{k L} \gamma^{\mu} u_{n L}\right)$ & $\begin{aligned} & \lambda_{i j}^{e} \lambda_{k n}^{u}\left[8 e^{2}\left(C_{1}-C_{3}\right)\right] \\
- & \lambda_{i j}^{e} \delta_{k n} \cdot \frac{8}{9} e^{2}\left[\left(C_{1}-3 C_{3}-C_{4}\right)-2\left(C_{1}-C_{3}\right)\left(\lambda_{33}^{u}+\hat{\lambda}_{22}^{u} \log \frac{m_{c}}{\mu}\right)\right. \\
& \left.+\left(\left(C_{1}+C_{3}\right) \hat{\lambda}_{33}^{d}+C_{4} \hat{\Gamma}_{33}^{d}\right) \log \frac{m_{b}}{\mu}\right] \\
+ & \delta_{i j} \lambda_{k n}^{u}\left[-\frac{4}{3} e^{2}\left(C_{1}-C_{3}+C_{6}\right)\right]\end{aligned}$ \\
\hline$\left(\bar{e}_{i L} \gamma_{\mu} e_{j L}\right)\left(\bar{u}_{k R} \gamma^{\mu} u_{n R}\right)$ & $\begin{aligned} &-\lambda_{i j}^{e} \delta_{k n} \cdot \frac{8}{9} e^{2}[\left(C_{1}-3 C_{3}-C_{4}\right)-2\left(C_{1}-C_{3}\right)\left(\lambda_{33}^{u}+\hat{\lambda}_{22}^{u} \log \frac{m_{c}}{\mu}\right) \\
&\left.+\left(\left(C_{1}+C_{3}\right) \hat{\lambda}_{33}^{d}+C_{4} \hat{\Gamma}_{33}^{d}\right) \log \frac{m_{b}}{\mu}\right]\end{aligned}$ \\
\hline$\left(\bar{e}_{i R} \gamma_{\mu} e_{j R}\right)\left(\bar{u}_{k L} \gamma^{\mu} u_{n L}\right)$ & $\begin{aligned} & \Gamma_{i j}^{e} \lambda_{k n}^{u}\left[-8 e^{2} C_{6}\right] \\
+ & \Gamma_{i j}^{e} \delta_{k n} \cdot \frac{8}{9} e^{2}\left[\left(C_{5}-C_{6}\right)+2 C_{6}\left(\lambda_{33}^{u}+\hat{\lambda}_{22}^{u} \log \frac{m_{c}}{\mu}\right)\right. \\
& \left.\quad-\left(C_{6} \hat{\lambda}_{33}^{d}+C_{5} \hat{\Gamma}_{33}^{d}\right) \log \frac{m_{b}}{\mu}\right] \\
+ & \delta_{i j} \lambda_{k n}^{u}\left[-\frac{4}{3} e^{2}\left(C_{1}-C_{3}+C_{6}\right)\right]\end{aligned}$ \\
\hline$\left(\bar{e}_{i R} \gamma_{\mu} e_{j R}\right)\left(\bar{u}_{k R} \gamma^{\mu} u_{n R}\right)$ & $\begin{aligned}+\Gamma_{i j}^{e} \delta_{k n} \cdot \frac{8}{9} e^{2} & {\left[\left(C_{5}-C_{6}\right)+2 C_{6}\left(\lambda_{33}^{u}+\hat{\lambda}_{22}^{u} \log \frac{m_{c}}{\mu}\right)\right.} \\
& \left.-\left(C_{6} \hat{\lambda}_{33}^{d}+C_{5} \hat{\Gamma}_{33}^{d}\right) \log \frac{m_{b}}{\mu}\right]\end{aligned}$ \\
\hline$\left(\bar{e}_{i L} \gamma_{\mu} e_{j L}\right)\left(\bar{d}_{k L} \gamma^{\mu} d_{n L}\right)$ & $\begin{aligned} & \lambda_{i j}^{e} \lambda_{k n}^{d}\left[-4 e^{2}\left(C_{1}+C_{3}\right)\right] \\
+ & \lambda_{i j}^{e} \delta_{k n} \cdot \frac{4}{9} e^{2}\left[\left(C_{1}-3 C_{3}-C_{4}\right)-2\left(C_{1}-C_{3}\right)\left(\lambda_{33}^{u}+\hat{\lambda}_{22}^{u} \log \frac{m_{c}}{\mu}\right)\right. \\
& \left.\quad+\left(\left(C_{1}+C_{3}\right) \hat{\lambda}_{33}^{d}+C_{4} \hat{\Gamma}_{33}^{d}\right) \log \frac{m_{b}}{\mu}\right] \\
+ & \delta_{i j} \lambda_{k n}^{d}\left[-\frac{4}{3} e^{2}\left(C_{1}+C_{3}+C_{6}\right)\right]\end{aligned}$ \\
\hline$\left(\bar{e}_{i L} \gamma_{\mu} e_{j L}\right)\left(\bar{d}_{k R} \gamma^{\mu} d_{n R}\right)$ & $\begin{aligned} & \lambda_{i j}^{e} \Gamma_{k n}^{d}\left[4 e^{2} C_{4}\right] \\
+ & \lambda_{i j}^{e} \delta_{k n} \cdot \frac{4}{9} e^{2}\left[\left(C_{1}-3 C_{3}-C_{4}\right)-2\left(C_{1}-C_{3}\right)\left(\lambda_{33}^{u}+\hat{\lambda}_{22}^{u} \log \frac{m_{c}}{\mu}\right)\right. \\
& \left.\quad+\left(\left(C_{1}+C_{3}\right) \hat{\lambda}_{33}^{d}+C_{4} \hat{\Gamma}_{33}^{d}\right) \log \frac{m_{b}}{\mu}\right] \\
+ & \delta_{i j} \Gamma_{k n}^{d} \cdot\left[-\frac{4}{3} e^{2}\left(C_{4}+C_{5}\right)\right]\end{aligned}$ \\
\hline$\left(\bar{e}_{i R} \gamma_{\mu} e_{j R}\right)\left(\bar{d}_{k L} \gamma^{\mu} d_{n L}\right)$ & $\begin{aligned} & \Gamma_{i j}^{e} \lambda_{k n}^{d}\left[4 e^{2} C_{6}\right] \\
+ & \Gamma_{i j}^{e} \delta_{k n} \cdot\left(-\frac{4}{9} e^{2}\right)\left[\left(C_{5}-C_{6}\right)+2 C_{6}\left(\lambda_{33}^{u}+\hat{\lambda}_{22}^{u} \log \frac{m_{c}}{\mu}\right)\right. \\
& \left.-\left(C_{6} \hat{\lambda}_{33}^{d}+C_{5} \hat{\Gamma}_{33}^{d}\right) \log \frac{m_{b}}{\mu}\right] \\
+ & \delta_{i j} \lambda_{k n}^{d}\left[-\frac{4}{3} e^{2}\left(C_{1}+C_{3}+C_{6}\right)\right]\end{aligned}$ \\
\hline$\left(\bar{e}_{i R} \gamma_{\mu} e_{j R}\right)\left(\bar{d}_{k R} \gamma^{\mu} d_{n R}\right)$ & $\begin{aligned} & \Gamma_{i j}^{e} \Gamma_{k n}^{d}\left[-4 e^{2} C_{5}\right] \\
+ & \Gamma_{i j}^{e} \delta_{k n} \cdot\left(-\frac{4}{9} e^{2}\right)\left[\left(C_{5}-C_{6}\right)+2 C_{6}\left(\lambda_{33}^{u}+\hat{\lambda}_{22}^{u} \log \frac{m_{c}}{\mu}\right)\right. \\
& \left.-\left(C_{6} \hat{\lambda}_{33}^{d}+C_{5} \hat{\Gamma}_{33}^{d}\right) \log \frac{m_{b}}{\mu}\right] \\
+ & \delta_{i j} \Gamma_{k n}^{d} \cdot\left[-\frac{4}{3} e^{2}\left(C_{4}+C_{5}\right)\right]\end{aligned}$ \\
\hline
\end{tabular}

Table 8. Operators $Q_{i}^{\mathrm{em}}$ and coefficients $\delta \xi_{i}$ for the semileptonic part of the effective Lagrangian $\delta \mathcal{L}_{\mathrm{eff}}^{\mathrm{QED}}$ involving charged leptons and neutral currents. For the down-type quarks generation indices run from 1 to 2 , while for up-type quarks we only keep the first generation. We set $\hat{\lambda}_{i i}^{u, d}=\lambda_{i i}^{u, d} / \log \frac{m_{\mathrm{EW}}}{\mu}$. 


\subsection{The $B$ anomalies}

The most significant measurements related to charged- and neutral-current B-anomalies are:

$$
\begin{aligned}
R_{D^{*}}^{\tau / \ell} & =\frac{\mathcal{B}\left(B \rightarrow D^{*} \tau \bar{\nu}\right)_{\exp } / \mathcal{B}\left(B \rightarrow D^{*} \tau \bar{\nu}\right)_{\mathrm{SM}}}{\mathcal{B}\left(B \rightarrow D^{*} \ell \bar{\nu}\right)_{\exp } / \mathcal{B}\left(B \rightarrow D^{*} \ell \bar{\nu}\right)_{\mathrm{SM}}}=1.23 \pm 0.07, \\
R_{D}^{\tau / \ell} & =\frac{\mathcal{B}(B \rightarrow D \tau \bar{\nu})_{\exp } / \mathcal{B}(B \rightarrow D \tau \bar{\nu})_{\mathrm{SM}}}{\mathcal{B}(B \rightarrow D \ell \bar{\nu})_{\exp } / \mathcal{B}(B \rightarrow D \ell \bar{\nu})_{\mathrm{SM}}}=1.34 \pm 0.17,
\end{aligned}
$$

where $\ell=e, \mu$, which follow from the HFAG averages [35] of Babar [1], Belle [3], and LHCb data [2], combined with the SM predictions [36, 37], and

$$
\begin{aligned}
& R_{K^{*}}^{\mu / e}=\left.\frac{\mathcal{B}\left(B \rightarrow K^{*} \mu \bar{\mu}\right)_{\exp }}{\mathcal{B}\left(B \rightarrow K^{*} e \bar{e}\right)_{\exp }}\right|_{q^{2} \in[1.1,6] \mathrm{GeV}}=0.685_{-0.069}^{+0.113} \pm 0.047 \\
& R_{K}^{\mu / e}=\left.\frac{\mathcal{B}(B \rightarrow K \mu \bar{\mu})_{\exp }}{\mathcal{B}(B \rightarrow K e \bar{e})_{\exp }}\right|_{q^{2} \in[1,6] \mathrm{GeV}}=0.745_{-0.074}^{+0.090} \pm 0.036
\end{aligned}
$$

based on combination of LHCb data [6] with the SM expectation $R_{K^{(*)}}^{\mu / e}=1.00 \pm 0.01[38,39]$.

We recall that $b \rightarrow s$ semileptonic transitions are conventionally described by means of the effective Lagrangian $\mathcal{L}_{\text {eff }}^{\mathrm{NC}}: 4$

$$
\mathcal{L}_{\text {eff }}^{\mathrm{NC}}=\frac{4 G_{F}}{\sqrt{2}} \lambda_{b s}^{t}\left(\mathcal{C}_{i j}^{9} \mathcal{O}_{i j}^{9}+\mathcal{C}_{i j}^{9^{\prime}} \mathcal{O}_{i j}^{9^{\prime}}+\mathcal{C}_{i j}^{10} \mathcal{O}_{i j}^{10}+\mathcal{C}_{i j}^{10^{\prime}} \mathcal{O}_{i j}^{10^{\prime}}+\mathcal{C}_{i j}^{\nu} \mathcal{O}_{i j}^{\nu}+\mathcal{C}_{i j}^{\nu^{\prime}} \mathcal{O}_{i j}^{\nu^{\prime}}\right)
$$

where $\lambda_{b s}^{t}=V_{t b} V_{t s}^{*}$ and the operators $\mathcal{O}^{i}$ are given by

$$
\begin{aligned}
\mathcal{O}_{i j}^{9} & =\frac{e^{2}}{(4 \pi)^{2}}\left(\bar{s} \gamma_{\mu} P_{L} b\right)\left(\bar{e}_{i} \gamma^{\mu} e_{j}\right), & \mathcal{O}_{i j}^{9^{\prime}} & =\frac{e^{2}}{(4 \pi)^{2}}\left(\bar{s} \gamma_{\mu} P_{R} b\right)\left(\bar{e}_{i} \gamma^{\mu} e_{j}\right), \\
\mathcal{O}_{i j}^{10} & =\frac{e^{2}}{(4 \pi)^{2}}\left(\bar{s} \gamma_{\mu} P_{L} b\right)\left(\bar{e}_{i} \gamma^{\mu} \gamma_{5} e_{j}\right), & \mathcal{O}_{i j}^{10^{\prime}} & =\frac{e^{2}}{(4 \pi)^{2}}\left(\bar{s} \gamma_{\mu} P_{R} b\right)\left(\bar{e}_{i} \gamma^{\mu} \gamma_{5} e_{j}\right), \\
\mathcal{O}_{i j}^{\nu} & =\frac{e^{2}}{(4 \pi)^{2}}\left(\bar{s} \gamma_{\mu} P_{L} b\right)\left(\bar{\nu}_{i} \gamma^{\mu}\left(1-\gamma_{5}\right) \nu_{j}\right), & \mathcal{O}_{i j}^{\nu^{\prime}} & =\frac{e^{2}}{(4 \pi)^{2}}\left(\bar{s} \gamma_{\mu} P_{R} b\right)\left(\bar{\nu}_{i} \gamma^{\mu}\left(1-\gamma_{5}\right) \nu_{j}\right)
\end{aligned}
$$

As to the charged-current transition $b \rightarrow c \ell \nu$, we address it using the effective Lagrangian $\mathcal{L}_{\text {eff }}^{\mathrm{CC}}$, defined as

$$
\mathcal{L}_{\text {eff }}^{\mathrm{CC}}=-\frac{4 G_{F}}{\sqrt{2}} \mathcal{C}_{\mathrm{L}}^{c b^{i j}}\left(\bar{c}_{L} \gamma^{\mu} b_{L}\right)\left(\bar{e}_{L i} \gamma_{\mu} \nu_{L j}\right)
$$

In our framework $B$ anomalies receive NP contributions at tree level. These contributions can be computed explicitly by matching the low-energy Lagrangians in eqs. (3.5), (3.7)

\footnotetext{
${ }^{4}$ In our analysis, the inclusion of dipole operators is not necessary as they provide negligible effects.
} 
with the NP Lagrangian $\mathcal{L}_{\mathrm{NP}}^{0} \cdot{ }^{5}$ As a result, we find

$$
\begin{array}{rlrl}
\left(\mathcal{C}_{\mathrm{NP}}^{9}\right)_{i j} & =\frac{4 \pi^{2}}{e^{2} \lambda_{b s}^{t}} \frac{v^{2}}{\Lambda^{2}} \lambda_{23}^{d}\left[\left(C_{1}+C_{3}\right) \lambda_{i j}^{e}+C_{6} \Gamma_{i j}^{e}\right] & \left(\mathcal{C}_{\mathrm{NP}}^{9^{\prime}}\right)_{i j}=\frac{4 \pi^{2}}{e^{2} \lambda_{b s}^{t}} \frac{v^{2}}{\Lambda^{2}} \Gamma_{23}^{d}\left[C_{4} \lambda_{i j}^{e}+C_{5} \Gamma_{i j}^{e}\right] \\
\left(\mathcal{C}_{\mathrm{NP}}^{10}\right)_{i j}=\frac{4 \pi^{2}}{e^{2} \lambda_{b s}^{t}} \frac{v^{2}}{\Lambda^{2}} \lambda_{23}^{d}\left[-\left(C_{1}+C_{3}\right) \lambda_{i j}^{e}+C_{6} \Gamma_{i j}^{e}\right] & \left(\mathcal{C}_{\mathrm{NP}}^{10^{\prime}}\right)_{i j}=\frac{4 \pi^{2}}{e^{2} \lambda_{b s}^{t}} \frac{v^{2}}{\Lambda^{2}} \Gamma_{23}^{d}\left[-C_{4} \lambda_{i j}^{e}+C_{5} \Gamma_{i j}^{e}\right] \\
\left(\mathcal{C}_{\mathrm{NP}}^{\nu}\right)_{i j}=\frac{4 \pi^{2}}{e^{2} \lambda_{b s}^{t}} \frac{v^{2}}{\Lambda^{2}} \lambda_{23}^{d} \lambda_{i j}^{e}\left(C_{1}-C_{3}\right) & \left(\mathcal{C}_{\mathrm{NP}}^{\nu^{\prime}}\right)_{i j}=\frac{4 \pi^{2}}{e^{2} \lambda_{b s}^{t}} \frac{v^{2}}{\Lambda^{2}} \Gamma_{23}^{d} \lambda_{i j}^{e} C_{4} \\
\left(\mathcal{C}_{L, \mathrm{NP}}^{c b}\right)_{i j}=-\frac{v^{2}}{\Lambda^{2}} \lambda_{23}^{u d} \lambda_{i j}^{e} C_{3}, &
\end{array}
$$

where subleading RGE terms have been neglected.

We remind that NP should contribute dominantly to charged-current transitions compared to the neutral-current ones, since in the SM the former arise at the tree-level while the latter at one-loop. This can be achieved in our framework by assuming a hierarchy between $\lambda_{33}^{d} \lambda_{33}^{e}$ and $\lambda_{23}^{d} \lambda_{22}^{e}$, which control $B \rightarrow D^{(*)} \tau \nu$ and $B \rightarrow K \mu^{+} \mu^{-}$, respectively.

\subsection{1 $\quad B \rightarrow K \ell \bar{\ell}$}

Keeping only linear terms in NP contributions, $R_{K^{(*)}}^{\mu / e}$ can be written in our framework as $[40,41]^{6}$

$$
\begin{aligned}
& R_{K}^{\mu / e} \simeq 1+0.24\left[\left(\mathcal{C}_{\mathrm{NP}}^{9}\right)_{\mu \mu}+\left(\mathcal{C}_{\mathrm{NP}}^{9^{\prime}}\right)_{\mu \mu}\right]-0.26\left[\left(\mathcal{C}_{\mathrm{NP}}^{10}\right)_{\mu \mu}+\left(\mathcal{C}_{\mathrm{NP}}^{10^{\prime}}\right)_{\mu \mu}\right] \\
& R_{K^{*}}^{\mu / e} \simeq 1+0.19\left[\left(\mathcal{C}_{\mathrm{NP}}^{9}\right)_{\mu \mu}-\left(\mathcal{C}_{\mathrm{NP}}^{9^{\prime}}\right)_{\mu \mu}\right]-0.29\left(\mathcal{C}_{\mathrm{NP}}^{10}\right)_{\mu \mu}+0.22\left(\mathcal{C}_{\mathrm{NP}}^{10^{\prime}}\right)_{\mu \mu}
\end{aligned}
$$

where $\left(\mathcal{C}_{\mathrm{NP}}^{9}\right)_{e e},\left(\mathcal{C}_{\mathrm{NP}}^{9^{\prime}}\right)_{e e},\left(\mathcal{C}_{\mathrm{NP}}^{10}\right)_{e e}$ and $\left(\mathcal{C}_{\mathrm{NP}}^{10^{\prime}}\right)_{e e}$ can be neglected because $\lambda_{11}^{e}=0$. Remembering that $\mathcal{C}_{\mathrm{SM}}^{9} \approx-\mathcal{C}_{\mathrm{SM}}^{10} \approx 4.2[42]$, we find the numerical expressions

$$
\begin{aligned}
& R_{K}^{\mu / e} \approx 1-\frac{0.30}{\Lambda^{2}\left(\mathrm{TeV}^{2}\right)} \frac{\lambda_{22}^{e}}{10^{-3}}\left[\left(C_{1}+C_{3}\right) \lambda_{23}^{d}+C_{4} \Gamma_{23}^{d}\right]+\cdots, \\
& R_{K^{*}}^{\mu / e} \approx 1-\frac{0.29}{\Lambda^{2}\left(\mathrm{TeV}^{2}\right)} \frac{1}{10^{-3}}\left[\left(C_{1}+C_{3}\right) \lambda_{22}^{e} \lambda_{23}^{d}-0.9 C_{4} \lambda_{22}^{e} \Gamma_{23}^{d}-0.2 C_{6} \lambda_{23}^{d} \Gamma_{22}^{e}\right]+\cdots
\end{aligned}
$$

and dots stand for smaller contributions. From (3.10) and the current experimental results (3.3) and (3.4), we argue that a simultaneous explanation of $R_{K}^{\mu / e}$ and $R_{K^{*}}^{\mu / e}$ requires the condition $\left|\left(C_{1}+C_{3}\right) \lambda_{23}^{d}\right| \gg\left|C_{4} \Gamma_{23}^{d}\right|$ and $\left(C_{1}+C_{3}\right) \lambda_{23}^{d} \lambda_{23}^{e} \approx \mathcal{O}\left(10^{-3}\right)$.

\subsection{2 $B \rightarrow D^{(*)} \ell \nu$}

LFUV in the charged-current process $B \rightarrow D^{(*)} \ell \nu$ is encoded in the observable $R_{D^{(*)}}^{\tau / \ell}$, which can be expressed as

$$
R_{D^{(*)}}^{\tau / \ell}=\frac{\sum_{j}\left|\left(\mathcal{C}_{L}^{c b}\right)_{3 j}\right|^{2}}{\sum_{j}\left|\left(\mathcal{C}_{L}^{c b}\right)_{\ell j}\right|^{2}} .
$$

\footnotetext{
${ }^{5}$ Strictly speaking $\mathcal{L}_{\text {eff }}^{\mathrm{NC}}$ and $\mathcal{L}_{\text {eff }}^{\mathrm{CC}}$ should be matched to the Lagrangian obtained by running the Wilson coefficients down to $\mu=m_{B}$, but RGE induced terms are generally negligible with respect to tree-level ones. This is true unless accidental cancellations among parameters take place, which we exclude.

${ }^{6}$ These expressions have been obtained using the Flavio code, see the website https://flav-io.github.io/.
} 
Keeping only linear NP contributions and neglecting $\lambda_{11}^{e}$ and $\lambda_{22}^{e}$ with respect to $\lambda_{33}^{e}$, we find

$$
R_{D^{(*)}}^{\tau / \ell} \approx 1-2 \frac{v^{2}}{\Lambda^{2}} \frac{\lambda_{23}^{u d}}{V_{c b}} C_{3} \lambda_{33}^{e}
$$

Then, using the relation $\lambda^{u d}=V_{\text {СKM }} \lambda^{d}$, we end up with the following expression

$$
R_{D^{(*)}}^{\tau / \ell} \approx 1-0.12 \frac{C_{3}}{\Lambda^{2}\left(\mathrm{TeV}^{2}\right)} \lambda_{33}^{e}\left(\frac{V_{c s}}{V_{c b}} \lambda_{23}^{d}+\lambda_{33}^{d}\right) .
$$

As a result, in order to accommodate the $R_{D^{(*)}}^{\tau / \ell}$ anomaly, we need $C_{3}<0$ and $C_{3} \sim \mathcal{O}(1)$, for $\Lambda=1 \mathrm{TeV}$.

\subsection{Tree-level semileptonic phenomenology}

Our framework predicts a set of deviations in leptonic and semileptonic $B$-decays which are strictly related to the anomalies discussed so far. Since dominant effects occur at tree level, the inclusion of quantum effects is not relevant here.

\subsection{1 $B \rightarrow \ell \nu$}

A charged-current process closely related to $B \rightarrow D^{(*)} \ell \nu$ is the decay $B \rightarrow \ell \nu$. We define the related LFUV observable, $R_{B \tau \nu}^{\tau / \ell}$, as

$$
R_{B \tau \nu}^{\tau / \mu}=\frac{\mathcal{B}(B \rightarrow \tau \nu)_{\exp } / \mathcal{B}(B \rightarrow \tau \nu)_{\mathrm{SM}}}{\mathcal{B}(B \rightarrow \mu \nu)_{\exp } / \mathcal{B}(B \rightarrow \mu \nu)_{\mathrm{SM}}} \approx 1-\frac{2 v^{2}}{\Lambda^{2}} C_{3} \lambda_{33}^{e}\left(\lambda_{33}^{d}+\frac{V_{u s} \lambda_{23}^{d} \cos \gamma}{\left|V_{u b}\right|}\right)
$$

where $\gamma \approx 70^{\circ}$. Since Belle II aims to measure $R_{B \tau \nu}^{\tau / \mu}$ with a $5 \%$ accuracy, it is likely that $R_{B \tau \nu}^{\tau / \mu}$ will provide a strong constraint to the present framework.

\subsection{2 $B \rightarrow K^{(*)} \nu \bar{\nu}$}

Another important process is $B \rightarrow K \bar{\nu} \nu$, which is strictly related to the neutral-current anomaly. We consider the observable $R_{K}^{\nu \nu}$, defined as

$$
R_{K}^{\nu \nu}=\frac{\mathcal{B}(B \rightarrow K \bar{\nu} \nu)}{\mathcal{B}(B \rightarrow K \bar{\nu} \nu)_{\mathrm{SM}}}
$$

which is subject to the experimental constraint $R_{K}^{\nu \nu}<4.3[43,44]$. In our framework $R_{K}^{\nu \nu}$ can be expressed as

$$
R_{K}^{\nu \nu}=\frac{\sum_{i j}\left|\mathcal{C}_{i j}^{\nu}+\mathcal{C}_{i j}^{\nu !}\right|^{2}}{3\left|\mathcal{C}_{\mathrm{SM}}^{\nu}\right|^{2}}=\frac{\sum_{i j}\left|\mathcal{C}_{\mathrm{SM}}^{\nu} \delta_{i j}+\left(\mathcal{C}_{\mathrm{NP}}^{\nu}\right)_{i j}+\left(\mathcal{C}_{\mathrm{NP}}^{\nu^{\prime}}\right)_{i j}\right|^{2}}{3\left|\mathcal{C}_{\mathrm{SM}}^{\nu}\right|^{2}}
$$

By expanding the numerator and using the property $\sum_{i j}\left|\lambda_{i j}^{e}\right|^{2}=1$ and $\sum_{i} \lambda_{i i}^{e}=1$, we find

$$
\begin{aligned}
R_{K}^{\nu \nu} \approx & +\frac{2}{3} \frac{\pi}{\alpha\left|\mathcal{C}_{\mathrm{SM}}^{\nu} \lambda_{b s}^{t}\right|} \frac{v^{2}}{\Lambda^{2}}\left[\left(C_{1}-C_{3}\right) \lambda_{23}^{d}+C_{4} \Gamma_{23}^{d}\right] \\
& +\frac{1}{3}\left(\frac{\pi}{\alpha\left|\mathcal{C}_{\mathrm{SM}}^{\nu} \lambda_{b s}^{t}\right|} \frac{v^{2}}{\Lambda^{2}}\left[\left(C_{1}-C_{3}\right) \lambda_{23}^{d}+C_{4} \Gamma_{23}^{d}\right]\right)^{2} .
\end{aligned}
$$


Since $\mathcal{C}_{\mathrm{SM}}^{\nu} \approx-6.4[44,45]$, we get the numerical result

$$
R_{K}^{\nu \nu} \approx 1+0.6\left(\frac{\lambda_{23}^{d}}{0.01} \frac{\left[\left(C_{1}-C_{3}\right)+C_{4} \Gamma_{23}^{d} / \lambda_{23}^{d}\right]}{\Lambda^{2}\left(\mathrm{TeV}^{2}\right)}\right)+0.3\left(\frac{\lambda_{23}^{d}}{0.01} \frac{\left[\left(C_{1}-C_{3}\right)+C_{4} \Gamma_{23}^{d} / \lambda_{23}^{d}\right]}{\Lambda^{2}\left(\mathrm{TeV}^{2}\right)}\right)^{2} .
$$

\subsection{3 $B_{s} \rightarrow \mu \bar{\mu}$}

NP contributions for the observable $R_{K}^{\mu / e}$ can also enter the process $B_{s} \rightarrow \mu \bar{\mu}$. In particular, NP effects for $B_{s} \rightarrow \mu \bar{\mu}$ are encoded by the following expression

$$
R_{B_{s} \mu \mu}=\frac{\mathcal{B}\left(B_{s} \rightarrow \mu \bar{\mu}\right)_{\mathrm{exp}}}{\mathcal{B}\left(B_{s} \rightarrow \mu \bar{\mu}\right)_{\mathrm{SM}}} \simeq\left|\frac{\mathcal{C}_{\mathrm{SM}}^{10}+\left(\mathcal{C}_{\mathrm{NP}}^{10}\right)_{\mu \mu}-\left(\mathcal{C}_{\mathrm{NP}}^{10^{\prime}}\right)_{\mu \mu}}{\mathcal{C}_{\mathrm{SM}}^{10}}\right|^{2}
$$

to be compared with the current experimental measurement and SM prediction for the branching ratio of this process $[46,47]$ :

$$
\mathcal{B}\left(B_{s} \rightarrow \mu \bar{\mu}\right)_{\exp }=2.8_{-0.6}^{+0.7} \times 10^{-9} \quad \mathcal{B}\left(B_{s} \rightarrow \mu \bar{\mu}\right)_{\mathrm{SM}}=3.65(23) \times 10^{-9} .
$$

\subsubsection{Lepton-flavour violating $B$ decays}

In our model, LFV decays like $B_{s} \rightarrow \tau^{ \pm} \mu^{\mp}$ and $B \rightarrow K \tau^{ \pm} \mu^{\mp}$ are generated at the tree level. Their branching ratios are given by [48]

$$
\begin{aligned}
\mathcal{B}\left(B_{s} \rightarrow \tau^{ \pm} \mu^{\mp}\right) & \approx 2 \times 10^{-8}\left(\left|\left(\mathcal{C}_{\mathrm{NP}}^{9}\right)_{\tau \mu}-\left(\mathcal{C}_{\mathrm{NP}}^{9^{\prime}}\right)_{\tau \mu}\right|^{2}+\left|\left(\mathcal{C}_{\mathrm{NP}}^{10}\right)_{\tau \mu}-\left(\mathcal{C}_{\mathrm{NP}}^{10^{\prime}}\right)_{\tau \mu}\right|^{2}\right) \\
\mathcal{B}\left(B \rightarrow K \tau^{ \pm} \mu^{\mp}\right) & \approx 2 \times 10^{-8}\left(\left|\left(\mathcal{C}_{\mathrm{NP}}^{9}\right)_{\tau \mu}+\left(\mathcal{C}_{\mathrm{NP}}^{9^{\prime}}\right)_{\tau \mu}\right|^{2}+\left|\left(\mathcal{C}_{\mathrm{NP}}^{10}\right)_{\tau \mu}+\left(\mathcal{C}_{\mathrm{NP}}^{10^{\prime}}\right)_{\tau \mu}\right|^{2}\right),
\end{aligned}
$$

where the factor of two in the above expressions accounts for the final state $\tau^{ \pm} \mu^{\mp}=$ $\tau^{+} \mu^{-}+\tau^{-} \mu^{+}$. As we will see shortly, loop-induced $\tau$ LFV decays are typically better probes of our scenario than LFV $B$-decays.

\subsection{One-loop phenomenology}

Electroweak corrections induce two main effects. First, $Z$ and $W$ couplings to fermions are modified with respect to the SM. Second, as we can see from eq. (2.18) and related tables, a purely leptonic Lagrangian is also generated at low energies. As a consequence, we expect LFV and LFUV effects in $Z, W$ and $\tau$ observables.

\subsubsection{Z-pole observables}

The NP modifications to $Z$ couplings arising in our setup, see eq. (2.15), explicitly break both LFV and LFUV. The consequent deviations of $Z$-pole observables from SM expectations are tightly constrained by LEP measurements of the $Z$ decay widths, left-right and forward-backward asymmetries. We recall the definition of the axial and vector couplings

$$
v_{\ell}=\left(g_{L}^{e}\right)_{\ell \ell}+\left(g_{R}^{e}\right)_{\ell \ell} \quad a_{\ell}=\left(g_{L}^{e}\right)_{\ell \ell}-\left(g_{R}^{e}\right)_{\ell \ell},
$$


and we consider the observables $v_{\tau} / v_{e}$ and $a_{\tau} / a_{e}$, which quantify the universality of $Z$ couplings to charged leptons. In our framework they read

$$
\begin{aligned}
& \frac{v_{\tau}}{v_{e}} \approx 1-\frac{2}{1-4 s_{\mathrm{W}}^{2}}\left[\left(\Delta g_{L}^{e}\right)_{33}-\left(\Delta g_{L}^{e}\right)_{11}+\left(\Delta g_{R}^{e}\right)_{33}-\left(\Delta g_{R}^{e}\right)_{11}\right] \\
& \frac{a_{\tau}}{a_{e}} \approx 1-2\left[\left(\Delta g_{L}^{e}\right)_{33}-\left(\Delta g_{L}^{e}\right)_{11}-\left(\Delta g_{R}^{e}\right)_{33}+\left(\Delta g_{R}^{e}\right)_{11}\right]
\end{aligned}
$$

leading to the following estimates

$$
\begin{aligned}
& \frac{v_{\tau}}{v_{e}} \approx 1-\frac{0.05}{\Lambda^{2}\left(\mathrm{TeV}^{2}\right)}\left[\left(C_{1}-C_{3}\right) \lambda_{33}^{e}+C_{6} \Gamma_{33}^{e}+0.2 C_{3} \lambda_{33}^{e}+0.02\left(\left(C_{1}-C_{4}\right) \lambda_{33}^{e}+\left(C_{6}-C_{5}\right) \Gamma_{33}^{e}\right)\right] \\
& \frac{a_{\tau}}{a_{e}} \approx 1-\frac{0.004}{\Lambda^{2}\left(\mathrm{TeV}^{2}\right)}\left[\left(C_{1}-C_{3}\right) \lambda_{33}^{e}-C_{6} \Gamma_{33}^{e}+0.2 C_{3} \lambda_{33}^{e}+0.02\left(\left(C_{1}-C_{4}\right) \lambda_{33}^{e}-\left(C_{6}-C_{5}\right) \Gamma_{33}^{e}\right)\right]
\end{aligned}
$$

to be compared with the LEP bounds [49]

$$
\frac{v_{\tau}}{v_{e}}=0.959(29) \quad \frac{a_{\tau}}{a_{e}}=1.0019(15) .
$$

Another important observable is the number of neutrinos $N_{\nu}$, which is extracted from the invisible $Z$ width. Taking the NP modification of $Z$ couplings to neutrinos into account, $N_{\nu}$ can be approximated by

$$
N_{\nu} \approx 3+4 \sum_{i}\left(\Delta g_{L}^{\nu}\right)_{i i} \approx 3+\frac{0.008}{\Lambda^{2}} \lambda_{33}^{e}\left[\left(C_{1}+C_{3}\right)-0.2 C_{3}+0.02\left(C_{1}-C_{4}\right)\right]
$$

while the experimental bound reads $N_{\nu}=2.9840 \pm 0.0082$ [49]. Electroweak quantum corrections give rise also to the LFV decay mode $Z \rightarrow \mu^{ \pm} \tau^{\mp}$. However, we have explicitly checked that in our model its branching ratio, typically of order $10^{-7}$, is always well below the current experimental bound $\mathcal{B}\left(Z \rightarrow \mu^{ \pm} \tau^{\mp}\right)_{\exp } \leq 1.2 \times 10^{-5}$. At the loop-level also the $W^{ \pm}$couplings to leptons are modified with respect to their SM expectations. However, the constraints on our model parameters arising from Z-pole observables are much stronger and therefore, hereafter, we neglect $W^{ \pm}$decays.

\subsubsection{Purely leptonic effective Lagrangian}

The effective low-energy Lagrangian (2.18) contains a purely leptonic Lagrangian $\mathcal{L}_{\text {eff }}^{\ell}$. Taking into account the explicit values of the $\xi_{i}$ and $\delta \xi_{i}$ for leptonic operators, and omitting terms manifestly respecting LFU in charged leptons, we can write it as

$$
\begin{gathered}
\mathcal{L}_{\mathrm{eff}}^{\ell}=-\frac{4 G_{F}}{\sqrt{2}}\left[\left(\bar{e}_{L} \gamma_{\mu} \lambda^{e} e_{L}\right) \sum_{f}\left(\bar{f} \gamma^{\mu} f\right)\left(2 g_{\mathrm{SM}}^{f} c_{t}^{e}-Q_{\psi} c_{\gamma}^{e}\right)+\left(\bar{e}_{R} \gamma_{\mu} \Gamma^{e} e_{R}\right) \sum_{f}\left(\bar{f} \gamma^{\mu} f\right)\left(2 g_{\mathrm{SM}}^{f} c_{t}^{e \prime}-Q_{f} c_{\gamma}^{e \prime}\right)\right. \\
\left.+c_{t}^{c c}\left(\bar{e}_{L} \gamma_{\mu} \lambda^{e} \nu_{L}\right)\left(\bar{\nu}_{L} \gamma_{\mu} e_{L}+\bar{u}_{L} \gamma_{\mu} V_{\mathrm{CKM}} d_{L}\right)+\text { h.c. }\right]
\end{gathered}
$$


where $f=\left\{\nu_{L}, e_{L}, e_{R}\right\}$ and $g_{\mathrm{SM}}^{f}$ is the $Z$ coupling to the $f$ field in the SM. The coefficients $c_{t}^{e}, c_{\gamma}^{e}, c_{t}^{e \prime}, c_{\gamma}^{e \prime}$ are given by

$$
\begin{aligned}
c_{t}^{e}= & \frac{3 v^{2} y_{t}^{2}}{32 \pi^{2} \Lambda^{2}}\left(C_{1}-C_{3}\right) \lambda_{33}^{u} \log \frac{\Lambda^{2}}{m_{\mathrm{EW}}^{2}} \\
c_{t}^{e^{\prime}}= & \frac{3 v^{2} y_{t}^{2}}{32 \pi^{2} \Lambda^{2}} C_{6} \lambda_{33}^{u} \log \frac{\Lambda^{2}}{m_{\mathrm{EW}}^{2}} \\
c_{\gamma}^{e}= & \frac{v^{2} e^{2}}{48 \pi^{2} \Lambda^{2}}\left[\left(3 C_{3}-C_{1}+C_{4}\right) \log \frac{\Lambda^{2}}{\mu^{2}}+2\left(C_{1}-C_{3}\right)\left(\lambda_{33}^{u} \log \frac{m_{\mathrm{EW}}^{2}}{\mu^{2}}+\lambda_{22}^{u} \log \frac{m_{c}^{2}}{\mu^{2}}\right)\right. \\
& \left.\quad-\left(\left(C_{1}+C_{3}\right) \lambda_{33}^{d}+C_{4} \Gamma_{33}^{d}\right) \log \frac{m_{b}^{2}}{\mu^{2}}\right] \\
c_{\gamma}^{e^{\prime}}= & \frac{v^{2} e^{2}}{48 \pi^{2} \Lambda^{2}}\left[\left(C_{6}-C_{5}\right) \log \frac{\Lambda^{2}}{\mu^{2}}+2 C_{6}\left(\lambda_{33}^{u} \log \frac{m_{\mathrm{EW}}^{2}}{\mu^{2}}+\lambda_{22}^{u} \log \frac{m_{c}^{2}}{\mu^{2}}\right)-\left(C_{6} \lambda_{33}^{d}+C_{5} \Gamma_{33}^{d}\right) \log \frac{m_{b}^{2}}{\mu^{2}}\right] \\
c_{t}^{c c}= & \frac{3 v^{2} y_{t}^{2}}{16 \pi^{2} \Lambda^{2}} C_{3} \lambda_{33}^{u} \log \frac{\Lambda^{2}}{m_{t}^{2}} .
\end{aligned}
$$

Notice that, in all observables analysed in this work but $R_{\tau}^{\tau / \ell_{1,2}}$ (see eq. (3.29)), we systematically neglected corrections to the Fermi constant. Their inclusion would amount to replace $G_{F}^{0}=v^{2} / \sqrt{2}$ with $G_{F}^{0} \simeq G_{F}\left(1-c_{t}^{c c} \lambda_{22}^{e}\right)$ where $G_{F}$ is the value extracted from the muon decay rate measurement. Numerically, such correction is below the $0.1 \%$ level and therefore safely negligible since $G_{F}^{0} \approx G_{F}\left(1-0.004 \lambda_{22}^{e} C_{3} / \Lambda^{2}(\mathrm{TeV})\right)$ with $\lambda_{22}^{e} \ll 1$.

Lagrangian (3.27) manifestly generates both LFV and LFUV processes. Given the hierarchy in $\lambda_{i j}^{e}$ and $\Gamma_{i j}^{e}$, NP effects are maximized in transitions involving the third generation. As a consequence, we focus on $\tau$ decays such as $\tau \rightarrow \ell \bar{\nu} \nu$ and $\tau \rightarrow 3 \mu$.

\subsection{3 $\tau \rightarrow \ell \bar{\nu} \nu$}

LFU breaking effects in $\tau \rightarrow \ell \bar{\nu} \nu$ (with $\ell_{1,2}=e, \mu$ ) are described by the observables

$$
R_{\tau}^{\tau / \ell_{1,2}}=\frac{\mathcal{B}\left(\tau \rightarrow \ell_{2,1} \nu \bar{\nu}\right)_{\exp } / \mathcal{B}\left(\tau \rightarrow \ell_{2,1} \nu \bar{\nu}\right)_{\mathrm{SM}}}{\mathcal{B}(\mu \rightarrow e \nu \bar{\nu})_{\exp } / \mathcal{B}(\mu \rightarrow e \nu \bar{\nu})_{\mathrm{SM}}}
$$

which are subject to the strong experimental constraints $R_{\tau}^{\tau / \mu}=1.0022 \pm 0.0030$ and $R_{\tau}^{\tau / e}=1.0060 \pm 0.0030$ [50]. Taking into account the correlation of these measurements, the combined constraint reads

$$
R_{\tau}^{\tau / \ell}=1.0032 \pm 0.0026
$$

In our setup the effective Lagrangian describing $e_{\alpha} \rightarrow e_{\beta} \bar{\nu}_{j} \nu_{i}$ is given by

$$
\mathcal{L}=-\frac{4 G_{F}}{\sqrt{2}}\left[\left(\mathcal{C}_{L}^{\alpha \beta}\right)_{i j}\left(\bar{e}_{\beta L} \gamma_{\mu} e_{\alpha L}\right)\left(\bar{\nu}_{i L} \gamma^{\mu} \nu_{j L}\right)+\left(\mathcal{C}_{R}^{\alpha \beta}\right)_{i j}\left(\bar{e}_{\beta R} \gamma_{\mu} e_{\alpha R}\right)\left(\bar{\nu}_{i L} \gamma^{\mu} \nu_{j L}\right)\right]
$$

where

$$
\begin{aligned}
& \left(\mathcal{C}_{L}^{\alpha \beta}\right)_{i j}=\delta_{\beta j} \delta_{i \alpha}+c_{t}^{e} \delta_{i j} \lambda_{\beta \alpha}^{e}+c_{t}^{c c}\left(\lambda_{\beta j}^{e} \delta_{i \alpha}+\lambda_{i \alpha}^{e} \delta_{\beta j}\right) \\
& \left(\mathcal{C}_{R}^{\alpha \beta}\right)_{i j}=c_{t}^{e^{\prime}} \Gamma_{\beta \alpha}^{e} \delta_{i j}
\end{aligned}
$$


Notice that the SM contribution to $e_{\alpha} \rightarrow e_{\beta} \bar{\nu}_{j} \nu_{i}$ is accounted for by the first term of (3.32). The ratio $R_{\tau}^{\tau / \ell}$ can be expressed in terms of these coefficients as follows

$$
R_{\tau}^{\tau / \ell_{1,2}}=\frac{\sum_{i j}\left|\left(\mathcal{C}_{L}^{\tau \ell_{2,1}}\right)_{i j}\right|^{2}+\left|\left(\mathcal{C}_{R}^{\tau \ell_{2,1}}\right)_{i j}\right|^{2}}{\sum_{i j}\left|\left(\mathcal{C}_{L}^{\mu e}\right)_{i j}\right|^{2}+\left|\left(\mathcal{C}_{R}^{\mu e}\right)_{i j}\right|^{2}} .
$$

Working linearly in the NP contribution, we find that

$$
\begin{aligned}
& R_{\tau}^{\tau / e} \simeq 1+2 c_{t}^{c c} \lambda_{33}^{e} \approx 1+0.008 \lambda_{33}^{e} \frac{C_{3}}{\Lambda^{2}(\mathrm{TeV})} \\
& R_{\tau}^{\tau / \mu} \simeq 1+2 c_{t}^{c c}\left(\lambda_{33}^{e}-\lambda_{22}^{e}\right) \approx 1+0.008\left(\lambda_{33}^{e}-\lambda_{22}^{e}\right) \frac{C_{3}}{\Lambda^{2}(\mathrm{TeV})}
\end{aligned}
$$

\subsection{4 $\tau \rightarrow 3 \mu$}

One of the most studied LFV processes generated by $\mathcal{L}_{\text {eff }}^{\ell}$ is the decay $\tau \rightarrow 3 \mu$, which is forbidden in the SM. The only contribution is given by $\mathcal{L}_{\text {eff }}^{\ell}$

$$
\begin{aligned}
\mathcal{L}_{\mathrm{eff}}^{\ell}=-\frac{4 G_{F}}{\sqrt{2}} & \left\{\lambda_{23}^{e}\left[\left(c_{L R}-c_{t}^{e}\right)\left(\mu_{L} \gamma_{\mu} \tau_{L}\right)\left(\bar{\mu}_{L} \gamma^{\mu} \mu_{L}\right)+c_{L R}\left(\mu_{L} \gamma_{\mu} \tau_{L}\right)\left(\bar{\mu}_{R} \gamma^{\mu} \mu_{R}\right)\right]\right. \\
& \left.+\Gamma_{23}^{e}\left[\left(c_{L R}^{\prime}-c_{t}^{e^{\prime}}\right)\left(\mu_{R} \gamma_{\mu} \tau_{R}\right)\left(\bar{\mu}_{L} \gamma^{\mu} \mu_{L}\right)+c_{L R}^{\prime}\left(\mu_{R} \gamma_{\mu} \tau_{R}\right)\left(\bar{\mu}_{R} \gamma^{\mu} \mu_{R}\right)\right]\right\}+\ldots
\end{aligned}
$$

where $c_{L R}^{(\prime)}=2 s_{\mathrm{w}}^{2} c_{t}^{e^{(\prime)}}+c_{\gamma}^{e^{(\prime)}}$. Adapting the formula given in ref. [51] we find

$$
\Gamma(\tau \rightarrow 3 \mu)=\frac{G_{F}^{2} m_{\tau}^{5}}{192 \pi^{3}}\left\{\left[2\left(c_{L R}-c_{t}^{e}\right)^{2}+c_{L R}^{2}\right]\left|\lambda_{23}^{e}\right|^{2}+\left[2 c_{L R}^{\prime 2}+\left(c_{L R}^{\prime}-c_{t}^{e^{\prime}}\right)^{2}\right]\left|\Gamma_{23}^{e}\right|^{2}\right\} .
$$

Keeping only the Yukawa contribution, which is typically the dominant one, we end up with the following numerical estimate

$$
\mathcal{B}(\tau \rightarrow 3 \mu) \approx\left(\frac{\lambda_{23}^{e}}{0.3}\right)^{2}\left[5.0 \frac{\left(C_{1}-C_{3}\right)^{2}}{\Lambda^{4}\left(\mathrm{TeV}^{4}\right)}+4.5 \frac{C_{6}^{2}}{\Lambda^{4}\left(\mathrm{TeV}^{4}\right)}\left(\frac{\Gamma_{23}^{e}}{\lambda_{23}^{e}}\right)^{2}\right] \cdot 10^{-8}
$$

to be compared with the current experimental bound $\mathcal{B}(\tau \rightarrow 3 \mu) \leqslant 1.2 \cdot 10^{-8}[35]$.

\subsection{Numerical analysis}

In this section, we focus on a phenomenologically relevant scenario where only $\left(\mathcal{C}_{\mathrm{NP}}^{9}\right)_{\mu \mu}$ is non-vanishing. This can be achieved by imposing the following conditions

$$
\Gamma_{i j}^{e}=\lambda_{i j}^{e} \quad C_{1}+C_{3}=C_{6} \quad C_{4}=C_{5}=0 .
$$

Taking the NP scale to be $\Lambda \approx 1 \mathrm{TeV}$, the free parameters in this setup are $C_{1}, C_{3}, \lambda_{23}^{d}$ and $\lambda_{23}^{e}$ where $\left|\lambda_{23}^{e, d}\right| \leq 0.5[23,24]$. We can further restrict the bounds on $\lambda_{23}^{e}$ because the non-observation of LFUV in $R_{D}^{\mu / e}$ implies that $\left|\lambda_{22}^{e}\right| \approx\left|\lambda_{23}^{e}\right|^{2} \leq 0.1$ [10]. As to $C_{1,3}$, 
we assume $\left|C_{1,3}\right| \leq 3$. Given (3.39), we obtain the following expressions for $B$-physics observables

$$
\begin{aligned}
R_{D^{(*)}}^{\tau / \ell} & =1-0.12 \frac{C_{3}}{\Lambda^{2}\left(\mathrm{TeV}^{2}\right)} \lambda_{33}^{e}\left(\frac{V_{c s}}{V_{c b}} \lambda_{23}^{d}+\lambda_{33}^{d}\right) \\
R_{K}^{\mu / e} & =1-\frac{0.30}{\Lambda^{2}\left(\mathrm{TeV}^{2}\right)} \frac{\lambda_{22}^{e} \lambda_{23}^{d}}{10^{-3}}\left(C_{1}+C_{3}\right) \\
R_{K^{*}}^{\mu / e} & =1-\frac{0.23}{\Lambda^{2}\left(\mathrm{TeV}^{2}\right)} \frac{\lambda_{22}^{e} \lambda_{23}^{d}}{10^{-3}}\left(C_{1}+C_{3}\right) \\
R_{K}^{\nu \nu} & =1+0.6\left(\frac{\lambda_{23}^{d}}{0.01} \frac{C_{1}-C_{3}}{\Lambda^{2}\left(\mathrm{TeV}^{2}\right)}\right)+0.3\left(\frac{\lambda_{23}^{d}}{0.01} \frac{C_{1}-C_{3}}{\Lambda^{2}\left(\mathrm{TeV}^{2}\right)}\right)^{2} .
\end{aligned}
$$

On the other hand, Z-pole observables simplify to

$$
\begin{aligned}
& \frac{v_{\tau}}{v_{e}}=1-\frac{0.10 \lambda_{33}^{e}}{\Lambda^{2}\left(\mathrm{TeV}^{2}\right)}\left(C_{1}+0.1 C_{3}\right) \\
& \frac{a_{\tau}}{a_{e}}=1+0.007 \lambda_{33}^{e} \frac{C_{3}}{\Lambda^{2}\left(\mathrm{TeV}^{2}\right)} \\
& N_{\nu}=3+\frac{0.008 \lambda_{33}^{e}}{\Lambda^{2}\left(\mathrm{TeV}^{2}\right)}\left(C_{1}+0.8 C_{3}\right) .
\end{aligned}
$$

Finally, for $\tau$ decays, we obtain the following estimates

$$
\begin{aligned}
R_{\tau}^{\tau / \ell_{1,2}} & =1+0.008 \lambda_{33}^{e} \frac{C_{3}}{\Lambda^{2}\left(\mathrm{TeV}^{2}\right)} \\
\mathcal{B}(\tau \rightarrow 3 \mu) & =\left(\frac{\lambda_{23}^{e}}{0.3}\right)^{2}\left[5.0 \frac{\left(C_{1}-C_{3}\right)^{2}}{\Lambda^{4}\left(\mathrm{TeV}^{4}\right)}+4.5 \frac{\left(C_{1}+C_{3}\right)^{2}}{\Lambda^{4}\left(\mathrm{TeV}^{4}\right)}\right] \cdot 10^{-8} .
\end{aligned}
$$

It is interesting to observe that the ratio $a_{\tau} / a_{e}$ depends exclusively on the Wilson coefficient $C_{3}$ of the charged-current operator. Choosing $\left|\lambda_{23}^{d}\right| \lesssim V_{c b}$ in order to avoid too much fine tuning when reproducing the CKM matrix, there is a strong correlation among $R_{D^{(*)}}^{\tau / \ell}$, $a_{\tau} / a_{e}$ and $R_{\tau}^{\tau / \ell_{1,2}}$. In particular, it turns out that the NP room left to $R_{D^{(*)}}^{\tau / \ell}$ is significantly reduced after taking into account all existing bounds. This can be clearly seen in the graph displayed in figure 1 , which shows the allowed regions for $R_{K}^{\mu / e}$ and $R_{D^{(*)}}^{\tau / \ell}$ after imposing the experimental bounds on Z-pole and $\tau$ observables at $2 \sigma$ level. ${ }^{7}$ Altough all observables receiving NP contribution at one loop impose strong bounds on $B$ anomalies, $Z$-pole observables set the stringest limits, forcing $\delta R_{D^{(*)}}^{\tau / \ell}$ to be $\lesssim 0.05$.

In the plot of figure 2 we analyse the correlation between the branching ratios of LFV decays, $B \rightarrow K \tau \mu$ and $\tau \rightarrow 3 \mu$. The graph shows that the loop-induced process $\tau \rightarrow 3 \mu$ is a much more sensitive probe of the considered scenario than the tree level observable $B \rightarrow K \tau \mu$, due to the current and expected future experimental resolution.

Finally, one could wonder which is the impact of the conditions of eq. (3.39) on our results. In order to answer this question, we made a general scan of the parameter space

\footnotetext{
${ }^{7}$ We do not show the plot in the $R_{K^{*}}^{\mu / e}$ vs. $R_{D^{(*)}}^{\tau / \ell}$ plane since it is almost indistinguishable to that of figure 1.
} 


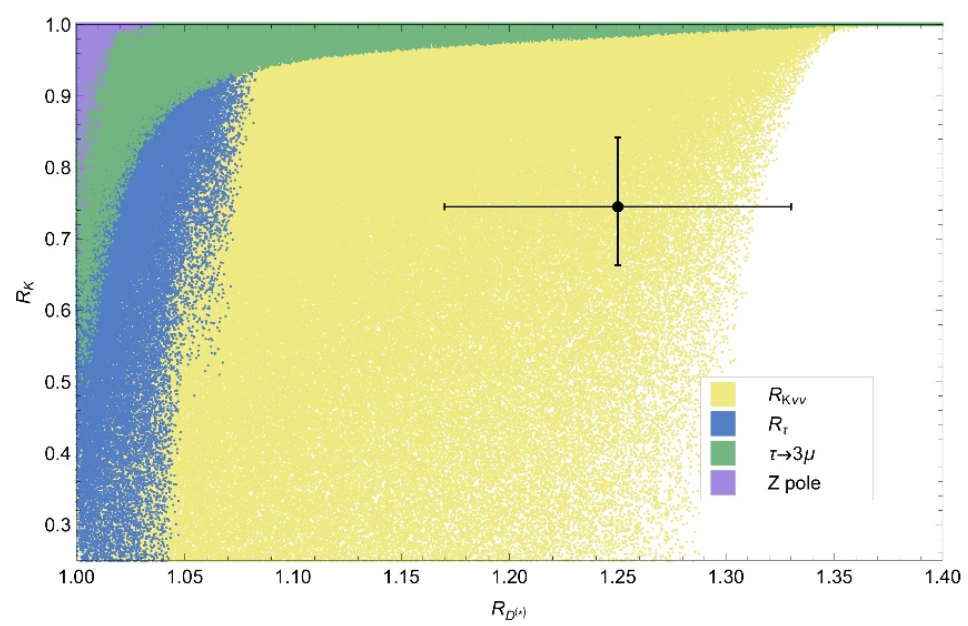

Figure 1. Impact of one-loop-induced constraints on the values of $R_{D^{(*)}}^{\tau / \ell}$ and $R_{K}^{\mu / e}$ for $C_{1} \in\{-3,3\}$, $C_{3} \in\{-3,3\}, \lambda_{23}^{e} \in\{-0.3,0.3\}$ and $\lambda_{23}^{d} \in\{-0.04,0.04\}$ and $\Lambda=1 \mathrm{TeV}$.

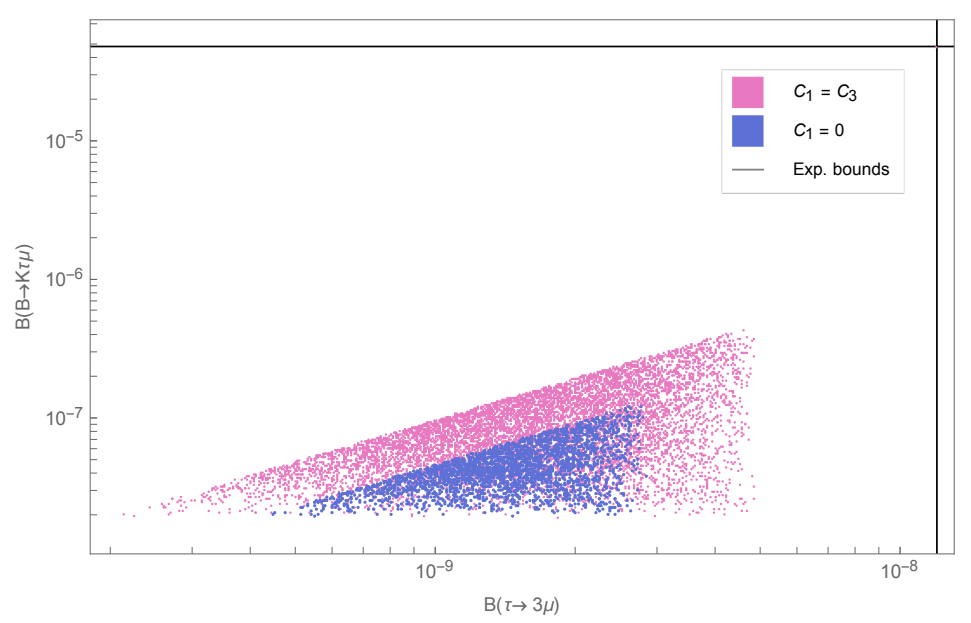

Figure 2. $\mathcal{B}(B \rightarrow 3 \mu)$ vs. $\mathcal{B}(B \rightarrow K \tau \mu)$ within our model for two different configurations of $C_{1}, C_{3}$, imposing all constraints but $R_{D^{(*)}}^{\tau / \ell}$. We let parameters vary in the ranges $C_{1} \in\{-3,3\}$, $C_{3} \in\{-3,3\}, \lambda_{23}^{e} \in\{-0.3,0.3\}$ and $\lambda_{23}^{d} \in\{-0.04,0.04\}$ and $\Lambda=1 \mathrm{TeV}$.

treating $\lambda_{23}^{e / d}, \Gamma_{23}^{e / d}$ and $C_{1-6}$ as independent parameters, while keeping couplings of NP to the third generation. The results of the numerical analysis is displayed in figure 3 . With respect to the scenario considered above, the additional freedom in the Wilson coefficients $C_{4}$, $C_{5}, C_{6}$ can lead to a partial cancellation of the NP contribution to $Z$-pole observables and $\tau \rightarrow 3 \mu$, hence relaxing significantly the corresponding experimental constraints. However, the tight bound from $R_{\tau}^{\tau / \ell}$ still prevents a simultaneous explanation of the charged-current anomalies $R_{D^{(*)}}^{\tau / \ell}$ and the neutral-current ones. This is due to the fact that $\lambda_{22}^{e}>0$ in our setup. Like in [23, 24], we therefore conclude that current data on $\tau$ and $Z$-pole observables challenge a simultaneous explanation of the present values of $R_{K^{(*)}}^{\mu / e}$ and $R_{D^{(*)}}^{\tau / \ell}$, when NP above the electroweak scale mainly affects the third generation. 


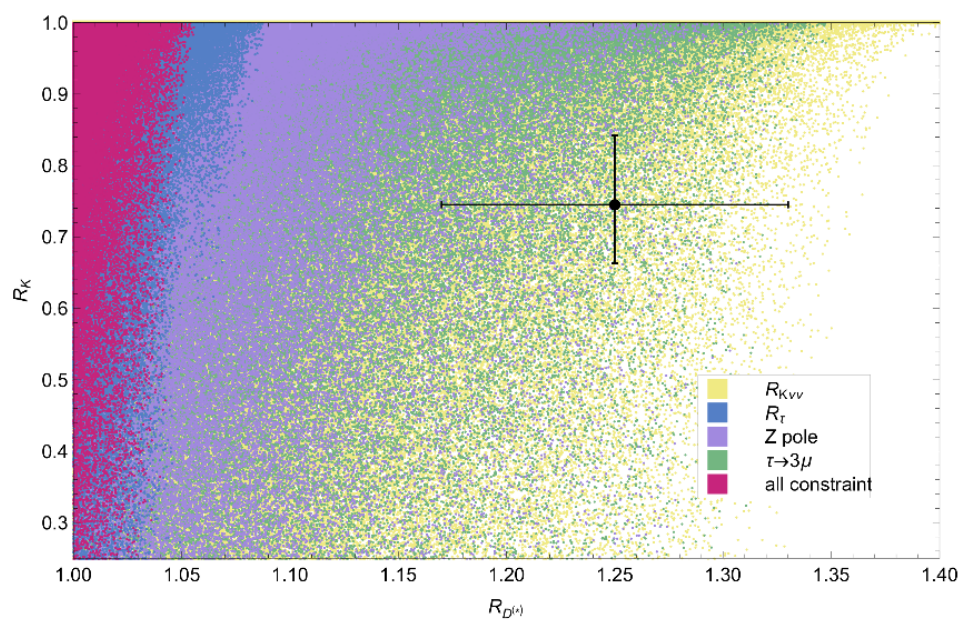

Figure 3. Impact of one-loop-induced constraints on the values of $R_{D^{(*)}}^{\tau / \ell}$ and $R_{K}^{\mu / e}$ for $C_{1-6} \in\{-3,3\}, \lambda_{23}^{d, e}$ and $\Gamma_{23}^{d, e} \in\{-0.3,0.3\}$, and $\Lambda=1 \mathrm{TeV}$.

The results of this section should not be interpreted as a no-go theorem for the simultaneous explanation of neutral and charged-current anomalies. We made use of a baseline scenario that, despite generalising that of refs. [23, 24], has clearly several limitations. In a UV complete model, purely leptonic four-fermion operators can be generated, providing additional contributions to the most constraining observables considered here. Cancellations between infrared logarithmic effects and boundary ultraviolet contributions cannot be excluded. Even though a complete compensation may sound as a fine tuning, a partial suppression of the effects discussed here can be sufficient to soften the bounds. Another limitation is related to the chosen flavour pattern. Dominant couplings of NP to the third generation is seriously motivated by both the experimental constraints involving the first two generations and by model building in the flavour sector, but should not be taken as a rule. Indeed, in the scenario with purely left-handed operators, it has been shown that relaxing this requirement allows to evade the bounds coming from the universality tests in $Z$ and $\tau$ decays [25]. On the one hand, a positive $\lambda_{23}^{d}$ of order 0.1 allows to raise the NP scale $\Lambda$ and decouple the radiative effects to a negligible level. In this case the solution to the neutral current anomalies requires $\lambda_{22}^{e}<0$, which is incompatible with the flavour pattern assumed here, but possible in a more general context. On the other hand, the new value of $\left|\lambda_{23}^{d}\right|$, much larger than the one considered here, can generate a tension in the phenomenology of $|\Delta F|=2$ transitions. These considerations remain valid also in the solution with the operator $O_{9}$ analysed here.

\section{Conclusions}

The persisting and coherent anomalous data in semileptonic B-decays point towards New Physics scenarios with large sources of Lepton Flavour Universality Violation. If this is the case, one would expect other non-standard effects to show up in low- and/or high-energy observables. The experimental signatures of specific scenarios able to accommodate these 
anomalies have been discussed extensively in the recent literature. On the other hand, the importance of including electroweak corrections in scenarios with left-handed semileptonic operators defined at the scale $\Lambda \gg v$ was stressed in refs. [23, 24].

In this work, by assuming that New Physics mainly affects the third generation, we have generalised the analysis of $[23,24]$ by considering an effective theory involving both purely left-handed operators $(V-A) \times(V-A)$ and operators with right-handed currents of the form $(V+A) \times(V+A)$ and $(V \pm A) \times(V \mp A)$. In this framework, we have derived the low-energy effective Lagrangian by means of the running and matching procedure outlined in [23, 24]. As in the previous analysis, we find that the dominant effects concern the corrections to the leptonic couplings of the $W$ and $Z$ vector bosons as well as the generation of a purely leptonic effective Lagrangian. Then we focused on a phenomenologically favoured setup where the dominant New Physics effects are encoded in the low-energy Wilson coefficient $C_{9}$ [7-9]. In this case we found that the strongest limit is posed by Z-pole observables. As shown by the analysis of the full parameter space, this constraint can be significantly relaxed by allowing for a more general New Physics structure. However, the bound from $R_{\tau}^{\tau / \ell}$ still prevents a simultaneous explanation of the anomalies. We therefore confirm and reinforce the conclusion that the stringent experimental bounds on Z-pole observables and $\tau$ decays severely reduce the New Physics room for a simultaneous explanation of charged and neutral-current non-standard data.

We stress that this results should not be interpreted as a no-go theorem. We adopted a baseline framework, which however assumes no conspiracy between UV and IR effects and dominance of the third generation in NP couplings. Relaxing these assumptions can allow to evade the bounds discussed here. Our main point is that the inclusion of electroweak corrections is mandatory to obtain reliable predictions.

\section{Acknowledgments}

We thank David Straub and Olcyr Sumensari for useful discussions. This work was supported in part by the MIUR-PRIN project 2010YJ2NYW and by the European Union network FP10 ITN ELUSIVES and INVISIBLES-PLUS (H2020-MSCA-ITN-2015-674896 and H2020-MSCA-RISE-2015-690575). The research of C.C. was supported in part by the Swiss National Science Foundation (SNF) under contract 200021-159720. The research of P.P. was supported in part by the ERC Advanced Grant No. 267985 (DaMeSyFla), by the research grant TAsP, and by the INFN. 
Open Access. This article is distributed under the terms of the Creative Commons Attribution License (CC-BY 4.0), which permits any use, distribution and reproduction in any medium, provided the original author(s) and source are credited.

\section{References}

[1] BABAR collaboration, J.P. Lees et al., Measurement of an Excess of $\bar{B} \rightarrow D^{(*)} \tau^{-} \bar{\nu}_{\tau}$ Decays and Implications for Charged Higgs Bosons, Phys. Rev. D 88 (2013) 072012 [arXiv: 1303.0571] [INSPIRE].

[2] LHCb collaboration, Measurement of the ratio of branching fractions $\mathcal{B}\left(\bar{B}^{0} \rightarrow D^{*+} \tau^{-} \bar{\nu}_{\tau}\right) / \mathcal{B}\left(\bar{B}^{0} \rightarrow D^{*+} \mu^{-} \bar{\nu}_{\mu}\right)$, Phys. Rev. Lett. 115 (2015) 111803 [Erratum ibid. 115 (2015) 159901] [arXiv: 1506.08614] [INSPIRE].

[3] Belle collaboration, S. Hirose et al., Measurement of the $\tau$ lepton polarization and $R\left(D^{*}\right)$ in the decay $\bar{B} \rightarrow D^{*} \tau^{-} \bar{\nu}_{\tau}$, Phys. Rev. Lett. 118 (2017) 211801 [arXiv:1612.00529] [INSPIRE].

[4] LHCb collaboration, Test of Lepton Flavor Universality by the measurement of the $B^{0} \rightarrow D^{*-} \tau^{+} \nu_{\tau}$ branching fraction using three-prong $\tau$ decays, Phys. Rev. D 97 (2018) 072013 [arXiv: 1711.02505] [INSPIRE].

[5] LHCb collaboration, Test of lepton universality using $B^{+} \rightarrow K^{+} \ell^{+} \ell^{-}$decays, Phys. Rev. Lett. 113 (2014) 151601 [arXiv: 1406.6482] [INSPIRE].

[6] LHCb collaboration, Test of lepton universality with $B^{0} \rightarrow K^{* 0} \ell^{+} \ell^{-}$decays, JHEP 08 (2017) 055 [arXiv: 1705. 05802] [INSPIRE].

[7] W. Altmannshofer and D.M. Straub, New physics in $b \rightarrow s$ transitions after LHC run 1, Eur. Phys. J. C 75 (2015) 382 [arXiv:1411.3161] [INSPIRE].

[8] S. Descotes-Genon, L. Hofer, J. Matias and J. Virto, Global analysis of $b \rightarrow$ sll anomalies, JHEP 06 (2016) 092 [arXiv: 1510.04239] [INSPIRE].

[9] G. Hiller and M. Schmaltz, $R_{K}$ and future $b \rightarrow$ sll physics beyond the standard model opportunities, Phys. Rev. D 90 (2014) 054014 [arXiv:1408.1627] [INSPIRE].

[10] A. Greljo, G. Isidori and D. Marzocca, On the breaking of Lepton Flavor Universality in B decays, JHEP 07 (2015) 142 [arXiv:1506.01705].

[11] A. Falkowski, M. Nardecchia and R. Ziegler, Lepton Flavor Non-Universality in B-meson Decays from a U(2) Flavor Model, JHEP 11 (2015) 173 [arXiv:1509.01249] [inSPIRE].

[12] R. Barbieri, G. Isidori, A. Pattori and F. Senia, Anomalies in B-decays and U(2) flavour symmetry, Eur. Phys. J. C 76 (2016) 67 [arXiv: 1512.01560] [InSPIRE].

[13] R. Barbieri, C.W. Murphy and F. Senia, B-decay Anomalies in a Composite Leptoquark Model, Eur. Phys. J. C 77 (2017) 8 [arXiv:1611.04930] [InSPIRE].

[14] L. Di Luzio, A. Greljo and M. Nardecchia, Gauge leptoquark as the origin of B-physics anomalies, Phys. Rev. D 96 (2017) 115011 [arXiv:1708.08450] [INSPIRE].

[15] L. Calibbi, A. Crivellin and T. Li, A model of vector leptoquarks in view of the B-physics anomalies, arXiv:1709.00692 [INSPIRE].

[16] M. Bordone, C. Cornella, J. Fuentes-Martin and G. Isidori, A three-site gauge model for flavor hierarchies and flavor anomalies, Phys. Lett. B 779 (2018) 317 [arXiv:1712.01368] [INSPIRE]. 
[17] R. Barbieri and A. Tesi, B-decay anomalies in Pati-Salam SU(4), Eur. Phys. J. C 78 (2018) 193 [arXiv: 1712.06844] [INSPIRE].

[18] D. Bečirević, S. Fajfer, N. Košnik and O. Sumensari, Leptoquark model to explain the B-physics anomalies, $R_{K}$ and $R_{D}$, Phys. Rev. D 94 (2016) 115021 [arXiv:1608.08501] [INSPIRE].

[19] M. Blanke and A. Crivellin, B Meson Anomalies in a Pati-Salam Model within the Randall-Sundrum Background, Phys. Rev. Lett. 121 (2018) 011801 [arXiv:1801.07256] [INSPIRE].

[20] B. Bhattacharya, A. Datta, D. London and S. Shivashankara, Simultaneous Explanation of the $R_{K}$ and $R\left(D^{(*)}\right)$ Puzzles, Phys. Lett. B 742 (2015) 370 [arXiv:1412.7164] [InSPIRE].

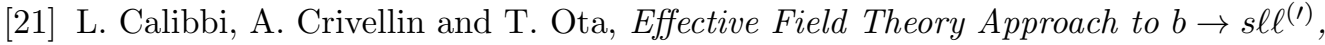
$B \rightarrow K^{(*)} \nu \bar{\nu}$ and $B \rightarrow D^{(*)} \tau \nu$ with Third Generation Couplings, Phys. Rev. Lett. 115 (2015) 181801 [arXiv: 1506.02661] [INSPIRE].

[22] S.L. Glashow, D. Guadagnoli and K. Lane, Lepton Flavor Violation in B Decays?, Phys. Rev. Lett. 114 (2015) 091801 [arXiv:1411.0565] [INSPIRE].

[23] F. Feruglio, P. Paradisi and A. Pattori, Revisiting Lepton Flavor Universality in B Decays, Phys. Rev. Lett. 118 (2017) 011801 [arXiv:1606.00524] [INSPIRE].

[24] F. Feruglio, P. Paradisi and A. Pattori, On the Importance of Electroweak Corrections for B Anomalies, JHEP 09 (2017) 061 [arXiv: 1705.00929] [INSPIRE].

[25] D. Buttazzo, A. Greljo, G. Isidori and D. Marzocca, B-physics anomalies: a guide to combined explanations, JHEP 11 (2017) 044 [arXiv: 1706.07808] [INSPIRE].

[26] D.A. Faroughy, A. Greljo and J.F. Kamenik, Confronting lepton flavor universality violation in B decays with high-p $p_{T}$ tau lepton searches at LHC, Phys. Lett. B 764 (2017) 126 [arXiv: 1609.07138] [INSPIRE].

[27] A. Greljo and D. Marzocca, High-p $p_{T}$ dilepton tails and flavor physics, Eur. Phys. J. C 77 (2017) 548 [arXiv : 1704.09015] [INSPIRE].

[28] R. Alonso, B. Grinstein and J. Martin Camalich, Lifetime of $B_{c}^{-}$Constrains Explanations for Anomalies in $B \rightarrow D^{(*)} \tau \nu$, Phys. Rev. Lett. 118 (2017) 081802 [arXiv:1611.06676] [INSPIRE].

[29] M. González-Alonso, J. Martin Camalich and K. Mimouni, Renormalization-group evolution of new physics contributions to (semi)leptonic meson decays, Phys. Lett. B 772 (2017) 777 [arXiv: 1706. 00410] [INSPIRE].

[30] D. Bardhan, P. Byakti and D. Ghosh, A closer look at the $R_{D}$ and $R_{D^{*}}$ anomalies, JHEP 01 (2017) 125 [arXiv: 1610.03038] [inSPIRE].

[31] D. Bardhan, P. Byakti and D. Ghosh, Role of Tensor operators in $R_{K}$ and $R_{K^{*}}$, Phys. Lett. B 773 (2017) 505 [arXiv:1705.09305] [INSPIRE].

[32] B. Grzadkowski, M. Iskrzynski, M. Misiak and J. Rosiek, Dimension-Six Terms in the Standard Model Lagrangian, JHEP 10 (2010) 085 [arXiv: 1008.4884] [INSPIRE].

[33] E.E. Jenkins, A.V. Manohar and M. Trott, Renormalization Group Evolution of the Standard Model Dimension Six Operators II: Yukawa Dependence, JHEP 01 (2014) 035 [arXiv:1310.4838] [INSPIRE]. 
[34] R. Alonso, E.E. Jenkins, A.V. Manohar and M. Trott, Renormalization Group Evolution of the Standard Model Dimension Six Operators III: Gauge Coupling Dependence and Phenomenology, JHEP 04 (2014) 159 [arXiv:1312.2014] [INSPIRE].

[35] HFLAV collaboration, Y. Amhis et al., Averages of b-hadron, c-hadron and $\tau$-lepton properties as of summer 2016, Eur. Phys. J. C 77 (2017) 895 [arXiv:1612.07233] [INSPIRE].

[36] S. Fajfer, J.F. Kamenik and I. Nisandzic, On the $B \rightarrow D^{*} \tau \bar{\nu}_{\tau}$ Sensitivity to New Physics, Phys. Rev. D 85 (2012) 094025 [arXiv:1203.2654] [InSPIRE].

[37] S. Aoki et al., Review of lattice results concerning low-energy particle physics, Eur. Phys. J. C 77 (2017) 112 [arXiv: 1607.00299] [INSPIRE].

[38] M. Bordone, G. Isidori and A. Pattori, On the Standard Model predictions for $R_{K}$ and $R_{K^{*}}$, Eur. Phys. J. C 76 (2016) 440 [arXiv: 1605.07633] [inSPIRE].

[39] G. Hiller and F. Krüger, More model-independent analysis of $b \rightarrow s$ processes, Phys. Rev. D 69 (2004) 074020 [hep-ph/0310219] [INSPIRE].

[40] G. Hiller and M. Schmaltz, Diagnosing lepton-nonuniversality in $b \rightarrow$ sll, JHEP 02 (2015) 055 [arXiv: 1411.4773] [INSPIRE].

[41] W. Altmannshofer, P. Stangl and D.M. Straub, Interpreting Hints for Lepton Flavor Universality Violation, Phys. Rev. D 96 (2017) 055008 [arXiv: 1704.05435] [INSPIRE].

[42] T. Blake, G. Lanfranchi and D.M. Straub, Rare B Decays as Tests of the Standard Model, Prog. Part. Nucl. Phys. 92 (2017) 50 [arXiv:1606.00916] [inSPIRE].

[43] BeLle collaboration, O. Lutz et al., Search for $B \rightarrow h^{(*)} \nu \bar{\nu}$ with the full Belle $\Upsilon(4 S)$ data sample, Phys. Rev. D 87 (2013) 111103 [arXiv:1303.3719] [INSPIRE].

[44] A.J. Buras, J. Girrbach-Noe, C. Niehoff and D.M. Straub, $B \rightarrow K^{(*)} \nu \bar{\nu}$ decays in the Standard Model and beyond, JHEP 02 (2015) 184 [arXiv:1409.4557] [INSPIRE].

[45] J. Brod, M. Gorbahn and E. Stamou, Two-Loop Electroweak Corrections for the $K \rightarrow \pi \nu \bar{\nu}$ Decays, Phys. Rev. D 83 (2011) 034030 [arXiv:1009.0947] [INSPIRE].

[46] LHCb and CMS collaborations, Observation of the rare $B_{s}^{0} \rightarrow \mu^{+} \mu^{-}$decay from the combined analysis of CMS and LHCb data, Nature $\mathbf{5 2 2}$ (2015) 68 [arXiv:1411.4413] [INSPIRE].

[47] C. Bobeth, M. Gorbahn, T. Hermann, M. Misiak, E. Stamou and M. Steinhauser, $B_{s, d} \rightarrow l^{+} l^{-}$in the Standard Model with Reduced Theoretical Uncertainty, Phys. Rev. Lett. 112 (2014) 101801 [arXiv:1311.0903] [INSPIRE].

[48] A. Crivellin, L. Hofer, J. Matias, U. Nierste, S. Pokorski and J. Rosiek, Lepton-flavour violating $B$ decays in generic $Z^{\prime}$ models, Phys. Rev. D 92 (2015) 054013 [arXiv: 1504.07928] [INSPIRE].

[49] Particle Data Group collaboration, K.A. Olive et al., Review of Particle Physics, Chin. Phys. C 38 (2014) 090001 [INSPIRE].

[50] A. Pich, Precision Tau Physics, Prog. Part. Nucl. Phys. 75 (2014) 41 [arXiv:1310.7922] [INSPIRE].

[51] Y. Kuno and Y. Okada, Muon decay and physics beyond the standard model, Rev. Mod. Phys. 73 (2001) 151 [hep-ph/9909265] [INSPIRE]. 